\title{
A Branch-Price-and-Cut Algorithm for the Capacitated Multiple Vehicle Traveling Purchaser Problem with Unitary Demand
}

\author{
Nicola Bianchessi*a,b, Stefan Irnich ${ }^{\mathrm{a}}$, Christian Tilk ${ }^{\mathrm{a}}$ \\ ${ }^{a}$ Chair of Logistics Management, Gutenberg School of Management and Economics, Johannes Gutenberg University, \\ Jakob-Welder-Weg 9, 55128 Mainz, Germany. \\ ${ }^{b}$ Dipartimento di Informatica, Università degli Studi di Milano, Via Celoria 18, I-20122 Milan, Italy.
}

\begin{abstract}
The multiple vehicle traveling purchaser problem (MVTPP) consists of simultaneously selecting suppliers and routing a fleet of homogeneous vehicles to purchase different products at the selected suppliers so that all product demands are fulfilled and traveling and purchasing costs are minimized. We consider variants of the MVTPP in which the capacity of the vehicles can become binding and the demand for each product is one unit. Corresponding solution algorithms from the literature are either branch-and-cut or branch-and-price algorithms, where in the latter case the route-generation subproblem is solved on an expanded graph by applying standard dynamic-programming techniques. Our branch-price-and-cut algorithm employs a novel labeling algorithm that works directly on the original network and postpones the purchasing decisions until the route has been completely defined. Moreover, we define a new branching rule generally applicable in case of unitary product demands, introduce a new family of valid inequalities to apply when suppliers can be visited at most once, and show how product incompatibilities can be handled without considering additional resources in the pricing problem. In comprehensive computational experiments with standard benchmark sets we prove that the new branch-price-and-cut approach is highly competitive.
\end{abstract}

Key words: vehicle routing, multiple vehicle traveling purchaser problem, unitary demand, incompatible products, column generation, dynamic-programming labeling algorithm

\section{Introduction}

In the context of procurement problems, the multiple vehicle traveling purchaser problem (MVTTP) can be seen as the problem faced by a company which needs to collect given amounts of different products from several suppliers. To this aim, the company operates a fleet of homogeneous vehicles. All vehicles are based at a common depot. Each supplier offers a subset of the products at possibly different prices (purchasing costs) and availabilities. The company has to select a subset of suppliers and construct a set of routes to visit them and collect products so that all product demands are fulfilled and traveling and purchasing costs are minimized.

Formally, MVTPP variants can be defined on a directed graph $G=(V, A)$ with vertex set $V=M \cup$ $\{0, m+1\}$ and arc set $A$. The vertices $M=\{1, \ldots, m\}$ represent the set of possible suppliers and vertices 0 and $m+1$ represent the depot where vehicle routes start and end, respectively. For each $\operatorname{arc}(i, j) \in A$ with $i \neq m+1$ and $j \neq 0$, the vehicles can travel from $i$ to $j$ with non-negative travel costs $c_{i j}$. In the following, it is assumed that the triangle inequality holds for the routing costs $\left(c_{i j}\right)$.

Let $K$ be the set of products to purchase. A non-negative quantity $q_{k i}$ of product $k \in K$ is available at supplier $i \in M$. There are no products available at the depot so that we can define $q_{k 0}=q_{k, m+1}=0$. Additionally, let $K_{i}=\left\{k \in K: q_{k i}>0\right\} \subseteq K$ be the subset of products sold by supplier $i \in M$, and let $M_{k}=\left\{i \in M: q_{k i}>0\right\} \subseteq M$ be the set of suppliers offering product $k \in K$. The non-negative purchasing costs of products $k$ at suppliers $i$ are denoted by $p_{k i}$. Moreover, for each product $k$ a positive demand $d_{k}$ has to be fulfilled, i.e., collected by one or several vehicles.

Suppliers are visited by means of a fleet $F$ of homogeneous vehicles each with capacity $Q$. In the following, a route $r$ is a pair of an elementary $0-(m+1)$-path in $G$ and positive quantities $\delta_{k i}^{r}$ of all products $k$

\footnotetext{
${ }^{*}$ Corresponding author.

Email address: nbianche@uni-mainz.de (Nicola Bianchessi)
} 
purchased at the visited suppliers $i$. The first component, the elementary $0-(m+1)$-path, can be described by $\left(i_{0}, i_{1}, i_{2}, \ldots, i_{L-1}, i_{L}\right)$ with the requirements that all vertices must be different, $i_{0}=0, i_{L}=m+1$, and $\left(i_{\ell-1}, i_{\ell}\right) \in A$ for all $\ell=1,2, \ldots, L$. A route $r$ is feasible if

(i) the total amount of products purchased at the visited suppliers does not exceed the vehicle capacity, i.e., $\sum_{\ell} \sum_{k} \delta_{k i_{\ell}}^{r} \leq Q$,

(ii) the amount of products $k$ purchased at suppliers $i_{\ell}$ does not exceed the available supply, i.e., $\delta_{k i_{\ell}}^{r} \leq$ $q_{k i_{\ell}}$ for all $\ell=1,2, \ldots, L$ and all $k \in K_{i_{\ell}}$, and

(iii) all purchases are feasible, i.e., $\delta_{k i_{\ell}}^{r}=0$ for all $\ell=1,2, \ldots, L$ and all $k \in K \backslash K_{i_{\ell}}$.

MVTPPs can be classified according to the following four categories referring to the available supply, the demand, the vehicle capacity, and the purchasing policy. First, the available supply can be

- restricted, if the available quantity is less than the product demand for at least one product available at a supplier;

- unrestricted, if the available quantity is not less than the corresponding demand for all products available at the suppliers.

Second, the demand can be classified as

- unitary, if the demand for all products is one;

- general, otherwise.

Third, the vehicles can be

- capacitated, meaning that the given capacity can become binding;

- uncapacitated, otherwise.

Fourth and finally, with respect to the purchasing policy we distinguish between

- split (purchases), if the same product can be purchased multiple times and more than one purchase can be smaller than the demand and the product availability at a supplier, i.e., $\delta_{k i}^{r}<\min \left\{d_{k}, q_{k i}\right\}$. To fulfill the demand of $k$, it can be that different suppliers are visited by the same vehicle or that several vehicles purchase product $k$ with visits to the same supplier or to different suppliers;

- non-split (purchases), otherwise. Note that, with restricted supply and general demand, also in this case it may be possible to visit different suppliers to fulfill the demand of a product, but according to this policy at most one purchase is allowed to be smaller than the demand and the product availability.

Table 1 summarizes the valid combinations for the four categories. Note that unitary demand implies an unrestricted supply and non-split purchases. Gray entries in a row of the table correspond to combinations giving rise to equivalent MVTPP variants. For a valid entry, there may still exist different MVTPP variants when additional side constraints are considered. One important class of side constraints is the single-visit constraints (SVC) imposing that no supplier is visited more than once. Without this requirement it is probably not possible to develop strong two-index compact models for the problem (for the related discussion on strong formulations for the split delivery vehicle-routing problem we refer to Bianchessi and Irnich, 2019). SVCs imply that all products that are available only at one specific supplier have to be purchased when this supplier is visited. Thus, in case of unitary demand and capacitated vehicles, imposing SVCs may cause the problem to become infeasible if the number of products that are available only at one specific supplier is greater than the capacity of the vehicles. Hence, when SVCs have to be fulfilled in this case, it is usually assumed that the problem remains feasible. Finally, it is worth noting that SVCs do not prevent split purchases, but impose that purchases of the same product must be done at different suppliers. In general, in case no further side constraint has to be considered, allowing or forbidding multiple visits to the same supplier is independent of the underlying purchasing policy.

\begin{tabular}{r|cccc|l} 
& non-split & non-split & split & split & \\
\hline unrestricted & {$[1,2,3,4,5,8]$} & & invalid & & capacited \\
unrestricted & {$[6]$} & & invalid & & uncapacited \\
restricted & invalid & & invalid & {$[2,7]$} & capacited \\
restricted & invalid & {$[9]$} & invalid & {$[6]$} & uncapacited \\
\hline & unitary & general & unitary & general &
\end{tabular}

Table 1: MVTPP variants — [1]: Baldacci et al. (2007); [2]: Choi and Lee (2010); [3]: Hoshino and De Souza (2012); [4]: Riera-Ledesma and Salazar-González (2012); [5]: Riera-Ledesma and Salazar-González (2013); [6]: Bianchessi et al. (2014); [7]: Manerba and Mansini (2015); [8]: Gendreau et al. (2016); [9]: Manerba and Mansini (2016).

The focus of this paper at hand is the unrestricted, capacitated, unitary MVTPP with non-split purchases 
(left-upper corner variant in Table 1). Before highlighting our contribution, we briefly review the MVTPP literature according to the above categories.

\subsection{Literature}

The MVTPP is NP-hard as it generalizes the well-known traveling purchaser problem (TPP, Manerba et al., 2017) to the multiple vehicles case. For an in-depth overview also on related procurement problems, the reader is referred to the cited survey. Here, we summarize the literature on the multi-vehicle case.

The MVTPP has been introduced by Choi and Lee (2010) who proposed Miller-Tucker-Zemlin formulations (Miller et al., 1960) for both the unrestricted and the restricted version, considering unitary and general demand, respectively, and capacitated vehicles. Instances with up to 40 suppliers, 40 products, and 4 vehicles or up to 30 suppliers, 30 products, and 3 vehicles were solved by means of CPLEX 11.1, respectively.

Riera-Ledesma and Salazar-González (2012) used the capacitated, unitary MVTPP to model a school bus routing problem. They presented a branch-and-cut (BC) algorithm based on a two-index single-commodity flow formulation, solving symmetric and asymmetric instances with up to 125 stops (suppliers), 125 students (products), and 6 buses (vehicles). Later, the same authors (Riera-Ledesma and Salazar-González, 2013) addressed an extended version of the problem considering upper bounds on the length/duration of each route and on the number of possible stops of each bus as well as lower bounds on the number of students served by each bus. The MVTTP formulation was augmented by additional constraints and finally solved with a branch-price-and-cut (BPC) algorithm. The pricing problem is modeled as an elementary shortest path problem with resource constraints (SPPRC, Irnich and Desaulniers, 2005) on an expanded graph, in which vertices correspond to all the potential assignments of a product to a supplier, and then solved through an adaptation of the q-routes dynamic-programming labeling algorithm (Christofides et al., 1981). Instances with up to 125 stops and 125 students were solved to optimality considering different combinations of active bounds.

Bianchessi et al. (2014) introduced unrestricted and restricted versions of the MVTPP that consider unitary and general demand, respectively. In both versions, the length of each route is bounded, whereas no limit is imposed on the capacity of the vehicles. The authors proposed a branch-and-price (BP) algorithm in which, thanks to the unlimited capacity of the vehicles, the pricing problem is directly modeled and solved as an elementary SPPRC on the original graph. The algorithm is able to solve instances with up to 100 suppliers, 200 products, and 8 vehicles.

Manerba and Mansini (2015) introduced a variant of the restricted, capacitated, general MVTPP, named MVTPP-PIC, involving possible incompatibilities among products that forbid to load two incompatible products into the same vehicle. Incompatibilities may require several visits to the same supplier, even if this is not beneficial with respect to traveling costs. Note that SVCs are typically not imposed for the MVTPP-PIC, because they may quickly lead to infeasibility. The problem was addressed by means of a BC algorithm that is based on a three-index formulation. The BC also makes use of an ad-hoc primal heuristic. The algorithm solves instances with up to 50 suppliers, 100 products, 20 percent of cross-incompatibilities among them, and 16 vehicles.

For the unitary demand version of the MVTPP-PIC, a BP algorithm was then presented by Gendreau et al. (2016). At each column generation iteration, the pricing problem is tentatively solved heuristically as an elementary SPPRC defined on an expanded graph as in (Riera-Ledesma and Salazar-González, 2013) by means of a labeling algorithm. To finally prove optimality, a BC algorithm is applied directly to a MIP formulation of the pricing problem resulting from the proposed Dantzig-Wolfe decomposition. The algorithm is able to solve instances up to 50 suppliers, 70 products, with 70 percent of cross-incompatibilities among them.

Finally, Manerba and Mansini (2016) introduced a further variant of the restricted, capacitated, general MVTPP to model a nurse routing problem with inter-route incompatibilities constraints and bounds on the duration of the routes. The BP algorithm proposed by the authors to solve the problem is similar to the one devised in Gendreau et al. (2016).

A closely related problem is the capacitated m-ring-star problem (CmRSP, Baldacci et al., 2007) that can also be modelled as a capacitated, unitary MVTPP. The CmRSP is the problem of designing a set of routes starting and ending at a given depot, visiting some customers in between, and assigning each non-visited customer to a visited point or customer. Baldacci et al. (2007) presented a sophisticated BC algorithm and solved instances with up to 137 customers to optimality. Hoshino and De Souza (2012) presented a BPC algorithm that solves the subproblem as an elementary SPPRC on a expanded graph using a complex 


\begin{tabular}{lccc}
\hline & $\begin{array}{c}\text { single-visit } \\
\text { constraints }\end{array}$ & $\begin{array}{c}\text { restricted } \\
\text { route length }\end{array}$ & $\begin{array}{c}\text { capacitated } \\
\text { with incom- } \\
\text { patibilities }\end{array}$ \\
\hline Baldacci et al. $(2007)$ & $\bullet$ & & $\bullet$ \\
Choi and Lee (2010) & $\bullet$ & & $\bullet$ \\
Hoshino and De Souza (2012) & $\bullet$ & & $\bullet$ \\
Riera-Ledesma and Salazar-González (2012) & $\bullet$ & $\bullet$ & $\bullet$ \\
Riera-Ledesma and Salazar-González (2013) & $\bullet$ & $\bullet$ & $\bullet$ \\
Bianchessi et al. $(2014)$ & $\bullet$ & $\bullet$ & $\bullet$ \\
Gendreau et al. $(2016)$ & & & $\bullet$ \\
\hline
\end{tabular}

Table 2: Characteristics of UMVTPP variants in the literature

dominance rule based on a deterministic finite automaton. Riera-Ledesma and Salazar-González (2012) used the instances of Baldacci et al. (2007) to computationally evaluate the performance of their algorithm.

Table 2 provides a synopsis of the unitary MVTPP (UMVTPP) variants that we surveyed. For the pairwise incompatibilities between different products, binary parameters $w_{k k^{\prime}}$ indicate whether products $k$ and $k^{\prime}$ are incompatible. The set of incompatible product pairs is denoted by $B=\left\{\left(k, k^{\prime}\right) \in K \times K: w_{k k^{\prime}}=1\right\}$ (see Manerba and Mansini, 2015; Gendreau et al., 2016). For the route duration constraints, non-negative travel times $d_{i j}$ are given, for all arcs $(i, j) \in A$, and the maximum route duration is denoted by $D^{\text {max }}$. A route is feasible if $\left(k, k^{\prime}\right) \notin B$ holds for all pairs of products $\left(k, k^{\prime}\right)$ purchased at the visited suppliers and the accumulated travel time $\sum_{\ell=1}^{L} d_{i_{\ell-1} i_{\ell}}$ for the path $\left(i_{0}, i_{1}, i_{2}, \ldots, i_{L-1}, i_{L}\right)$ does not exceed $D^{\text {max }}$.

\subsection{Contribution}

The overall contribution of the paper at hand is the presentation of a new BPC algorithm that can deal with the basic version of the capacitated UMVTPP considering additional SVCs (as previously addressed by five of the nine articles reviewed, see Table 1 and 2), as well as with the extensions arising from incompatibility constraints and route duration constraints. Particular contributions are:

1. All previous BP algorithms for the capacitated UMVTPP or one of its extensions solve the routegeneration subproblem on an expanded graph with standard dynamic-programming labeling algorithms (Riera-Ledesma and Salazar-González, 2013; Gendreau et al., 2016) or BC algorithms (Gendreau et al., 2016; Manerba and Mansini, 2016). Our new labeling algorithm works directly on the original network, i.e., the one with vertices for the suppliers and the depot. The key idea of the new approach is to postpone the purchasing decision until the route has been completely defined. On the positive side, this drastically reduces the size of the pricing network and thus accelerating the solution process. On the downside, new and more complex dominance rules have to be defined.

2. For the UMVTPP and its extensions, we introduce a new generally applicable branching rule.

3. We introduce a new family of valid inequalities for strengthening SVCs.

4. Finally, we show how incompatibilities can be handled without additional resources in the pricing problem by considering multiple pricing problems.

Our later presented computational results indicate that the new subproblem solution strategies are beneficial, especially for instances in which many products are available at a supplier and routes are relatively short.

The remainder of this paper is organized as follows: Section 2 describes our BPC algorithm with subsections on the extensive formulation, the pricing subproblem, valid inequalities, and branching. In Section 3, we computationally evaluate the BPC using instances from different benchmarks. Final conclusions are drawn in Section 4.

\section{Branch-Price-and-Cut Algorithm}

This section describes the new BPC algorithm for the solution of different unitary MVTPP variants. Section 2.1 presents the straightforward set-partitioning formulation that provides the basis for the columngeneration master program. The route-generation subproblem is discussed in Section 2.2. The valid inequalities used to strengthen the linear relaxation of the master program and a dynamic neighborhood extension are presented in Sections 2.3 and 2.4. Branching rules to finally obtain integer solutions are discussed in Section 2.5 . 


\subsection{Route-based Formulation}

We now propose a general route-based formulation that can model all the addressed unitary MVTPP variants, and on which our BPC algorithm is based on. Recall that a route $r$ is defined as an elementary 0 - $(m+1)$-path together with the purchasing decisions $\delta_{k i}^{r}$ for the products $k \in K$ offered by the suppliers $i \in M$ visited en route. Let $\Omega$ denote the set of all feasible routes. Feasibility takes into account all variantspecific intra-route constraints as described in Section 1. For a route $r \in \Omega$, let the binary coefficient $b_{i j}^{r}$ gives the number of times that arc $(i, j) \in A$ is traversed ( 0 or 1 for elementary routes). Finally, the cost of the route is defined as $c^{r}=\sum_{(i, j) \in A} c_{i j} b_{i j}^{r}+\sum_{k \in K} \sum_{i \in M_{k}} p_{k i} \delta_{k i}^{r}$ including routing and purchasing costs.

The route-based formulation uses two types of variables: Binary variables $\lambda^{r}$ for all $r \in \Omega$ are equal to 1 if route $r$ is performed. The non-negative integer variable $f$ describes the number of vehicles used.

$$
\begin{aligned}
\min & \sum_{r \in \Omega} c^{r} \lambda^{r} \\
\text { subject to } & \sum_{r \in \Omega} \sum_{i \in M_{k}} \delta_{k i}^{r} \lambda^{r}=1 \\
& \sum_{r \in \Omega} \lambda^{r}=f \\
& 0 \leq f \leq|F| \text { and integer } \\
& \lambda^{r} \in\{0,1\}
\end{aligned}
$$

The objective (1a) calls for the minimization of the total traveling and purchasing costs. The set-partitioning constraints (1b) ensure that all products are purchased exactly once. Constraints (1c) and (1d) limit the number of vehicles to use. Binary constraints (1e) are stated for all route variables.

In the following, the linear relaxation of formulation (1) in which the set of all feasible routes $\Omega$ is replaced by a subset $\bar{\Omega}$ is denoted as restricted master program (RMP). We initialize the RMP with one big- $M$ variable that covers all product demands and has no impact on other constraints. For solving the linear relaxation of (1), a column-generation algorithm is employed (Desaulniers et al., 2005). Branching is required to finally ensure integer solutions.

\subsection{Column Generation}

In this section, we consider an arbitrary column-generation iteration and thus assume that the dual prices $\left(\rho_{k}\right)_{k \in K}$ associated with constraints (1b) and the dual price $\mu$ of constraint (1c) are given.

We define the reduced purchasing cost $\bar{p}_{k i}$ of product $k \in K$ at supplier $i \in M$ as $\bar{p}_{k i}=p_{k i}-\rho_{k}$. The pricing problem asks for a route $r$ with negative reduced cost

$$
\bar{c}_{r}=c_{r}-\sum_{k \in K} \sum_{i \in M_{k}} \rho_{k} \delta_{k i}^{r}-\mu=\sum_{(i, j) \in A} c_{i j} b_{i j}^{r}+\sum_{k \in K} \sum_{i \in M_{k}} \bar{p}_{k i} \delta_{k i}^{r}-\mu .
$$

For the sake of clarity, we first state this pricing problem as a binary program. We use the standard notation $A(S)=\{(i, j) \in A: i \in S, j \in S\}$ for the internal, $\Gamma^{+}(S)=\{(i, j) \in A: i \in S, j \notin S\}$ the outgoing, and $\Gamma^{-}(S)=\{(i, j) \in A: i \notin S, j \in S\}$ the ingoing arcs of a vertex set $S \subset V$. For singleton sets $S=\{i\}$, we write $\Gamma^{+}(i)$ and $\Gamma^{-}(i)$. The binary program used to find a negative reduced cost route $r$, defined by an elementary path and purchased quantities, comprises binary variables $x_{i j}$ and $z_{i}$ that are equal to 1 if and only if $\operatorname{arc}(i, j) \in A$ is traversed and supplier $i \in M$ is visited, respectively. Moreover, binary variables $\delta_{k i}$ 
indicate if product $k \in K$ is purchased at supplier $i \in M_{k}$. The subproblem can now be stated as follows:

$$
\begin{aligned}
\min & -\mu+\sum_{(i, j) \in A} c_{i j} x_{i j}+\sum_{k \in K} \sum_{i \in M_{k}} \bar{p}_{k i} \delta_{k i} \\
\text { subject to } & \sum_{i \in M} x_{0 i}=1 \\
& \sum_{(j, i) \in \Gamma^{-}(i)} x_{j i}=\sum_{(i, j) \in \Gamma^{+}(i)} x_{i j}=z_{i} \\
& \sum_{(i, j) \in \Gamma^{+}(S)} x_{i j} \geq z_{i} \\
& \sum_{k \in K} \sum_{i \in M_{k}} \delta_{k i} \leq Q \\
& \delta_{k i} \leq z_{i} \\
& \delta_{k i} \in\{0,1\} \\
& z_{i} \in\{0,1\} \\
& x_{i j} \in\{0,1\}
\end{aligned}
$$

The objective (2a) is the minimization of the reduced cost. Constraints $(2 \mathrm{~b})-(2 \mathrm{~d})$ together with $(2 \mathrm{~h})$ ensure the route to be an elementary $0-(m+1)$-path. Inequality $(2 \mathrm{e})$ imposes the capacity constraint. Consistency between variables $\delta_{k i}$ and $z_{i}$ is ensured by (2f). Binary domains for all variables are stated by $(2 \mathrm{~g})-(2 \mathrm{i})$.

Property 1. If the triangle inequality for travel costs $\left(c_{i j}\right)$ holds, then there exists an optimal solution to the UMVTPP in which at least one product is purchased at every visited supplier $i \in M$.

Proof. See (Riera-Ledesma and Salazar-González, 2012).

For each supplier $i \in M$, let $K_{i}^{*}=\left\{k \in K_{i}:\{i\}=M_{k}\right\}$ denote the set of unique products that can only be purchased at supplier $i$. Assuming that the routing costs fulfill the triangle inequality (as stated in Section 1), we can strengthen formulation (2) of the pricing problem by

$$
\begin{array}{lr}
\sum_{i \in M} z_{i} \leq Q & \\
\sum_{k \in K_{i}} \delta_{k i} \geq z_{i} & i \in M \\
\delta_{k i}=z_{i} & i \in M, k \in K_{i}^{*}
\end{array}
$$

where constraint (3a) implies that the number of visited suppliers does not exceed the vehicle capacity, constraints (3b) guarantee at least one purchase per visited supplier, and constraints (3c) guarantee that all products that are only available at supplier $i$ are bought when this supplier is visited.

\subsubsection{Dynamic-Programming Labeling Algorithm}

We now present the new labeling algorithm that works directly on the original network. The handling of side constraints concerning single visits, incompatibilities, and the maximum route duration is discussed in Section 2.2.2.

The pricing problem (2) is a variant of the SPPRC, for which dynamic programming-based labeling algorithms are commonly used in BP algorithms when solving vehicle-routing and crew-scheduling problems (Irnich and Desaulniers, 2005). In simple SPPRC variants, resource consumptions along arcs are known. In MVTPP variants, however, the products to purchase at each visited supplier are decision variables, yielding different reduced costs and resource consumptions. To circumvent additional decisions per arc traversed, the MVTPP can be modeled over an expanded graph, in which vertices correspond to all potential combinations of a product and a supplier (see, e.g., Riera-Ledesma and Salazar-González, 2013; Gendreau et al., 2016). This modeling approach drastically increases the size of the pricing network. Hence, previous works using the expanded network may suffer from long computation times required for pricing.

Our idea of solving the pricing problem directly on the original network strives for accelerated pricing using relatively small networks. Moreover, we postpone all unclear decisions concerning the potential purchase of products at visited suppliers until the end of the route, i.e., when reaching the destination depot $m+1$. 
We show first how to compute the smallest reduced purchasing cost when a path is given. Let a partial path $P=\left(0, i_{1}, \ldots, i_{n}\right)$ be given, i.e., a path that does not necessarily end at the destination $m+1$. For simplification purposes, we introduce the shorthand notations $S(P)=M \cap V(P)=\left\{i_{1}, \ldots, i_{n}\right\}$ for the set of visited suppliers and $K(P)=K_{i_{1}} \cup K_{i_{2}} \cup \cdots \cup K_{i_{n}}$ for the set of products that can be purchased on path $P$. For each product $k \in K(P)$, we define the minimum reduced purchasing cost (MRPC) given by

$$
\bar{p}_{k}^{*}(S(P))=\min _{i \in S(P)} \bar{p}_{k i}
$$

If the context is clear, we may lighten the notation and write $\bar{p}_{k}^{*}$ instead of $\bar{p}_{k}^{*}(S(P))$.

Note first that only those products that have a strictly negative MRPC are relevant for an optimal purchasing decision. Let $h$ be the number of these products so that the $h$ products can be sorted by nondecreasing MRPCs. Accordingly, we define

$$
O(S(P))=\left(\left(k_{1}, \bar{p}_{k_{1}}^{*}\right), \ldots,\left(k_{h}, \bar{p}_{k_{h}}^{*}\right)\right)
$$

as the sorted sequence of pairs of products and their negative MRPCs. Note that absolute values are nonincreasing, i.e., $\left|\bar{p}_{k_{1}}^{*}\right| \geq\left|\bar{p}_{k_{2}}^{*}\right| \geq \ldots \geq\left|\bar{p}_{k_{h}}^{*}\right|$.

It is optimal to purchase the first $\bar{h}=\min \{Q,|O(S(P))|\}=\min \{Q, h\}$ products $k_{1}, k_{2}, \ldots, k_{\bar{h}}$ of the sequence. As $Q$ is constant, the overall reduced purchasing cost can now be described by the function

$$
f(S(P))=\sum_{q=1}^{\bar{h}} \bar{p}_{k_{q}}^{*}
$$

As a result, the minimum reduced cost of any route $r$ using path $P=\left(0=i_{0}, i_{1}, \ldots, i_{L}, i_{L+1}=m+1\right)$ with routing cost $c(P)=\sum_{\ell=1}^{L+1} c_{i_{\ell-1}, i_{\ell}}$ is

$$
\bar{c}_{r}=c(P)+f(S(P))-\mu
$$

Note that such a sequence and function can also be defined independently of a path $P$. One only has to know the subset of suppliers (for the path $P$ the suppliers $S=S(P)$ ). Therefore, for any subset $S \subseteq M$ of suppliers, we define $O(S)$ and $f(S)$ accordingly via (4a)-(4c).

Proposition 1. The function $f: 2^{M} \rightarrow \mathbb{R}, S \mapsto f(S)$ defined by (4a)-(4c) has the following properties:

(i) $f$ is non-positive, i.e., $f(S) \leq 0$ for all $\varnothing \subseteq S \subseteq M$;

(ii) $f$ is non-increasing, i.e., for any $R \subseteq S \subseteq M$ it follows $f(R) \geq f(S)$;

(iii) $f$ is supermodular ( $f$ is submodular), i.e., for any $R, S \subseteq M$ it follows $f(R)+f(S) \leq f(R \cup S)+$ $f(R \cap S)$.

Proof. (i): By definition of $f$ because all values $\bar{p}_{k_{q}}^{*}$ are negative for $q=1, \ldots, \bar{h}$.

(ii): $R \subseteq S$ implies that the minima fulfill $\bar{p}_{k_{q}}^{*}(R) \geq \bar{p}_{k_{q}}^{*}(S)$. Hence, the sequence $O(R)$ may contain less elements compared to $O(S)$ (this happens if $\left.K(R)=\bigcup_{i \in R} K_{i} \subsetneq K(S)=\bigcup_{i \in S} K_{i}\right)$. Moreover, for elements in the sequences with identical first component, i.e., $\left(k, \bar{p}_{k}^{*}(R)\right) \in O(R)$ and $\left(k, \bar{p}_{k}^{*}(S)\right) \in O(S)$, it follows $\bar{p}_{k}^{*}(R) \geq \bar{p}_{k}^{*}(S)$. Hence, $\bar{h}(R) \leq \bar{h}(S)$ and therefore $f(R) \geq f(S)$ holds true.

(iii): To prove the submodularity of $-f$ it is equivalent to prove that for any $S \subseteq S^{\prime}$ and $i \in M$ the inequality

$$
f(S)-f(S \cup\{i\}) \geq f\left(S^{\prime}\right)-f\left(S^{\prime} \cup\{i\}\right)
$$

holds (see, e.g., Schrijver, 2003, $\S 44$, p. 766).

Note that the only interesting cases are those when $i \notin S^{\prime}$, because otherwise $S^{\prime}=S^{\prime} \cup\{i\}$ so that the right-hand side in (6) is zero and the statement directly follows then from part (ii), i.e., since $f$ is non-increasing.

We can therefore assume that $i \notin S^{\prime}$ holds. If $f\left(S^{\prime}\right)=f\left(S^{\prime} \cup\{i\}\right)$, then (6) follows directly from part (ii). We can therefore assume that $f\left(S^{\prime}\right)>f\left(S^{\prime} \cup\{i\}\right)$ holds.

To simplify the analysis, we assume the sequences $O(S)$ always comprise $Q$ entries. This is not restrictive because shorter sequences can be filled up with dummy products $k_{\text {dummy }}$ with $\mathrm{MRPC} \bar{p}_{k_{\text {dummy }}}=0$. In particular, the $Q$ th element of $O(S)$ is then the product with non-positive but worst MRPC compared to all other products in the sequence $O(S)$.

Moreover, it is convenient to write $k \in O(S)$ if and only if there exists a pair $\left(k_{h}, \bar{p}_{k_{h}}^{*}\right)$ in the sequence $O(S)$ with $k=k_{h}$ for some index $h \in\{1, \ldots, Q\}$. We use this convention in the following. 
Another simplification is that we assume (for the moment) that $K_{i}=\{k\}$ holds, i.e., the supplier $i$ offers a single product $k$ only. Then, due to $f\left(S^{\prime}\right)>f\left(S^{\prime} \cup\{i\}\right)$,

$$
k \in O\left(S^{\prime} \cup\{i\}\right) \text { with } p_{k i}=\bar{p}_{k}^{*}\left(S^{\prime} \cup\{i\}\right) .
$$

Moreover, $S \subseteq S^{\prime}$ implies that

$$
k \in O(S \cup\{i\}) \text { with } p_{k i}=\bar{p}_{k}^{*}(S \cup\{i\}) .
$$

We can now distinguish four cases:

- Case $k \in O(S)$ and $k \in O\left(S^{\prime}\right)$ : Note first that the precondition of this case implies, together with (7) and (8), that both $O(S)$ and $O(S \cup\{i\})$ as well as $O\left(S^{\prime}\right)$ and $O\left(S^{\prime} \cup\{i\}\right)$ differ in exactly one pair, i.e., the pair $\left(k_{h}, \bar{p}_{k_{h}}^{*}\right)$ with $k_{h}=k$ has differing values $\bar{p}_{k_{h}}^{*}$. Therefore,

$$
f(S)-f(S \cup\{i\}) \stackrel{(8)}{=} \bar{p}_{k}^{*}(S)-p_{k i} \stackrel{S \subseteq S^{\prime}}{\geq} \bar{p}_{k}^{*}\left(S^{\prime}\right)-p_{k i} \stackrel{(7)}{=} f\left(S^{\prime}\right)-f\left(S^{\prime} \cup\{i\}\right) .
$$

Note that the inequality directly results from the definition of $\bar{p}_{k}^{*}(\cdot)$ as a minimum of the subsets $K(S) \subseteq$ $K\left(S^{\prime}\right)$, respectively, see equation (4a).

- Case $k \notin O(S)$ and $k \in O\left(S^{\prime}\right)$ : The precondition of this case, together with $S \subseteq S^{\prime}$, implies that $\bar{p}_{k}^{*}\left(S^{\prime}\right) \leq$ $\bar{p}_{k_{Q}}^{*}(S)$, where $k_{Q}$ is the product of the last pair $\left(k_{Q}, \bar{p}_{k_{Q}}^{*}\right)$ in the sequence $O(S)$. According to $(8), O(S \cup\{i\})$ results from $O(S)$ by removing its last pair $\left(k_{Q}, \bar{p}_{k_{Q}}^{*}\right)$ and inserting the pair $\left(k, p_{k i}\right)$. It follows

$$
f(S)-f(S \cup\{i\}) \stackrel{(8)}{=} \bar{p}_{k_{Q}}^{*}(S)-p_{k i} \stackrel{k \notin O(S) \wedge k \in O\left(S^{\prime}\right)}{\geq} \bar{p}_{k}^{*}\left(S^{\prime}\right)-p_{k i} \stackrel{(7)}{=} f\left(S^{\prime}\right)-f\left(S^{\prime} \cup\{i\}\right) .
$$

- Case $k \in O(S)$ and $k \notin O\left(S^{\prime}\right)$ : With arguments similar to those from the previous case we get

$$
f(S)-f(S \cup\{i\}) \stackrel{(8)}{=} \bar{p}_{k}^{*}(S)-p_{k i} \stackrel{k \in O(S) \wedge k \notin O\left(S^{\prime}\right)}{\geq} \bar{p}_{k_{Q}}^{*}\left(S^{\prime}\right)-p_{k i} \stackrel{(7)}{=} f\left(S^{\prime}\right)-f\left(S^{\prime} \cup\{i\}\right) .
$$

- Case $k \notin O(S)$ and $k \notin O\left(S^{\prime}\right)$ : With the arguments from the two previous cases, the remaining last case is solved via

$$
f(S)-f(S \cup\{i\}) \stackrel{(8)}{=} \bar{p}_{k_{Q}}^{*}(S)-p_{k i} \stackrel{S \subseteq S^{\prime}}{\geq} \bar{p}_{k_{Q}}^{*}\left(S^{\prime}\right)-p_{k i} \stackrel{(7)}{=} f\left(S^{\prime}\right)-f\left(S^{\prime} \cup\{i\}\right) .
$$

What remains to discuss is the case that supplier $i$ offers more than just one product, i.e., $\left|K_{i}\right|>1$. If $K_{i}=\left\{k_{1}, k_{2}, \ldots, k_{q}\right\}$ for $q \geq 2$, we can, just for the purpose of the proof, replace the one supplier $i$ by $q$ different copies named suppliers $i_{1}, i_{2}, \ldots, i_{q}$ with the product sets $K_{i_{j}}=\left\{k_{j}\right\}$. These copies are singleproduct suppliers for which the above-discussed four cases hold true. With $f(S \cup\{i\})=f\left(S \cup\left\{i_{1}, i_{2}, \ldots, i_{q}\right\}\right)$ and likewise $f\left(S^{\prime} \cup\{i\}\right)=f\left(S^{\prime} \cup\left\{i_{1}, i_{2}, \ldots, i_{q}\right\}\right)$ we get

$$
\begin{aligned}
f(S)-f(S \cup\{i\}) & =f(S)-f\left(S \cup\left\{i_{1}, i_{2}, \ldots, i_{q}\right\}\right) \\
& \left.=(f(S\})-f\left(S \cup\left\{i_{1}\right\}\right)\right)+\sum_{j=2}^{q}\left(f\left(S \cup\left\{i_{1}, \ldots, i_{j-1}\right\}\right)-f\left(S \cup\left\{i_{1}, i_{2}, \ldots, i_{j-1}, i_{j}\right\}\right)\right) \\
& \left.\geq\left(f\left(S^{\prime}\right\}\right)-f\left(S^{\prime} \cup\left\{i_{1}\right\}\right)\right)+\sum_{j=2}^{q}\left(f\left(S^{\prime} \cup\left\{i_{1}, \ldots, i_{j-1}\right\}\right)-f\left(S^{\prime} \cup\left\{i_{1}, i_{2}, \ldots, i_{j-1}, i_{j}\right\}\right)\right) \\
& =f\left(S^{\prime}\right)-f\left(S^{\prime} \cup\left\{i_{1}, i_{2}, \ldots, i_{q}\right\}\right)=f\left(S^{\prime}\right)-f\left(S^{\prime} \cup\{i\}\right)
\end{aligned}
$$

where the inequality results from the four cases discussed above for single-product suppliers. This completes the proof.

Forward Labeling. Let $P$ be the partial path starting at the depot 0 and ending at vertex $i \in V$. We associate a label $L$ with $P$ (to highlight the association we write $L=L(P)$ and reversely $P=P(L)$ ) that includes the following attributes:

$i$ : The last vertex of $P$;

$S$ : The set $S(P)$ of visited suppliers;

$T^{\text {cost }}$ : The accumulated routing costs $c(P)$ including also (parts of) the value $\mu$. 
$T^{\text {num }}:$ The (minimum) number of collected products;

$O$ : The sequence of pairs (product, MRPC value), available at the visited suppliers, sorted by non-decreasing MRPC values as defined in (4b), i.e., $O(S(P))=O(S)$.

Note that the sequence $O$ can be computed via (4a) and (4b) directly from the subset $S$. We store the sequence $O$ within the label for ease of convenience and speed (one can directly see which products are bought at which visited supplier). Hence, for any partial path $P$, we can write the associated label $L=$ $L(P)=(i, S, T)$, where $T$ is the vector of attributes $\left(T^{\text {cost }}, T^{\text {num }}\right)$. Later, this latter vector will be extended to take additional constraints into account and to implement acceleration techniques.

We can now describe the labeling in more detail, i.e., the initial label, label extension, feasibility conditions, and dominance between labels. The initial label at vertex 0 is defined as $L_{0}=(0, \varnothing, \mathbf{0})$.

When a feasible path $P=(0, \ldots, i)$ with associated label $L_{i}=\left(i, S_{i}, T_{i}\right)$ is extended along arc $(i, j) \in A$, the new label $L_{j}=\left(j, S_{j}, T_{j}\right)$ for $P^{\prime}=(0, \ldots, i, j)$ results from the following update rules:

$$
\begin{aligned}
S_{j} & = \begin{cases}S_{i} \cup\{j\}, & \text { if } j \in M \\
S_{i} & \text { otherwise }\end{cases} \\
T_{j}^{\text {cost }} & =T_{i}^{\text {cost }}+c_{i j}- \begin{cases}\mu / 2, & \text { if } i=0 \text { or } j=m+1 \\
0, & \text { otherwise }\end{cases} \\
T_{j}^{\text {num }} & =T_{i}^{\text {num }}+ \begin{cases}1, & \text { if } j \in M \\
0, & \text { otherwise }\end{cases}
\end{aligned}
$$

The (partial) path $P^{\prime}$ is feasible if

$$
T_{j}^{\text {num }} \leq Q
$$

This feasibility condition is a direct consequence of Property 1 exploiting (3a).

Note that up to now we did not require the paths to be elementary. Indeed, with the following dominance rule all non-elementary paths are dominated by their cycle-free subpaths whenever the triangle inequality for the routing costs $\left(c_{i j}\right)$ holds.

Proposition 2. Let label $L_{1}=\left(i, S_{1}, T_{1}\right)$ and label $L_{2}=\left(i, S_{2}, T_{2}\right)$ be two labels that represent two different paths, i.e., $P\left(L_{1}\right) \neq P\left(L_{2}\right)$, that end at the same vertex $i \in V$.

Label $L_{1}$ dominates label $L_{2}$ if the following conditions both hold true:

$$
\begin{aligned}
T_{1}^{\text {cost }}+f\left(S_{1}\right) & \leq T_{2}^{\text {cost }}+f\left(S_{1} \cup S_{2}\right) \\
T_{1}^{\text {num }} & \leq T_{2}^{\text {num }}
\end{aligned}
$$

Proof. Note first that any feasible extension of $P_{2}=P\left(L_{2}\right)$ along a path $\bar{P}$ is certainly also a feasible extension of $P_{1}=P\left(L_{1}\right)$ because of $(11 \mathrm{~b})$ and the feasibility condition (10).

We assume that the extension along path $\bar{P}$ visits the suppliers $\bar{S}$. We have to show that inequality (11a) implies

$$
T_{1}^{\text {cost }}+c(\bar{P})+f\left(S_{1} \cup \bar{S}\right) \leq T_{2}^{\text {cost }}+c(\bar{P})+f\left(S_{1} \cup S_{2} \cup \bar{S}\right)
$$

which is equivalent to the implication

$$
T_{1}^{\text {cost }}-T_{2}^{\text {cost }} \leq f\left(S_{1} \cup S_{2} \cup \bar{S}\right)-f\left(S_{1} \cup \bar{S}\right) .
$$

We write

$$
\begin{aligned}
T_{1}^{\text {cost }}-T_{2}^{\text {cost }} & \stackrel{(11 \mathrm{a})}{\leq} \\
& \stackrel{(6)}{\leq} \quad f\left(S_{1} \cup S_{2}\right)-f\left(S_{1}\right) \\
& f\left(S_{1} \cup S_{2} \cup \bar{S}\right)-f\left(S_{1} \cup \bar{S}\right)
\end{aligned}
$$

where the last inequality holds for $S_{1}=S \subseteq S^{\prime}=S_{1} \cup S_{2}$ and $\bar{S} \equiv\{i\}$, with $K_{i}=K(\bar{P})$ arbitrary large. This completes the proof.

If a path $P_{2}$ is not elementary, the removal of suppliers visited two or more times (from the top) creates an elementary path $P_{1}$ ending at the same vertex $i$. Now $P_{1}$ with label $L_{1}=\left(i, S_{1}, T_{1}\right)$ dominates $P_{2}$ with label $L_{2}=\left(i, S_{2}, T_{2}\right)$, because the triangle inequality for the routing costs ensures $T_{1}^{\text {cost }} \leq T_{2}^{\text {cost }}$ and by construction $T_{1}^{\text {num }}<T_{2}^{\text {num }}$ as well as $S_{1}=S_{2}$ so that both conditions (11) are fulfilled. 
ng-Path Relaxation. Cutting planes (Section 2.3) and branching constraints (Section 2.5) introduce additional dual prices on arcs that can make the associated reduced cost negative. In this situation, negative reduced-cost cycles can occur so that non-elementary partial paths are no longer dominated by elementary paths constructed by leaving out suppliers that are visited multiple times. It is known for a long time that the linear relaxation of path-based formulations can be strengthened by eliminating all or at least some nonelementary routes and their variables from the model. In this case, the pricing subproblem must prohibit the generation of non-elementary partial paths.

Baldacci et al. (2011) invented $n g$-path relaxations, parameterized by neighborhoods $N_{i} \subset M$ for all $i \in V$, to effectively eliminate many non-elementary paths. For given neighborhoods $N_{i}$, we enforce $n g$-path constraints for the UMVTPP as follows. Each label $L=(j, S, T)$ is complemented by binary attributes $T^{n g, k}$ for all suppliers $k \in N_{j}$. The initial label at vertex 0 has attributes $T^{n g}=\mathbf{0}$. When extending a partial path $P_{i}$ associated with label $L_{i}=L\left(P_{i}\right)$ along the $\operatorname{arc}(i, j) \in A, j \in N_{i}$, the additional feasibility condition $T_{i}^{n g, j}=0$ is checked. If the extension is feasible the new attribute is updated via $T_{j}^{n g, j}=1$ and $T_{j}^{n g, k}=T_{i}^{n g, k}$ if $k \in N_{j} \cap N_{i}$, and $T_{j}^{n g, k}=0$ otherwise. Finally, the dominance rules (11) have to be supplemented by the (componentwise) check $T_{1}^{n g} \leq T_{2}^{n g}$ when comparing two labels $L_{1}=\left(i, S_{1}, T_{1}\right)$ and $L_{2}=\left(i, S_{2}, T_{2}\right)$. Section 2.4 explains the strategy that we apply to build initial neighborhoods $N_{i}$ and how we enlarge the neighborhoods in the course of the algorithm in order to strengthen the linear relaxations.

Bidirectional Labeling. Bidirectional labeling has become a quasi-standard for solving SPPRCs (Righini and Salani, 2006; Tilk et al., 2017). Defining backward labels for the UMVTPP is trivial, because one can swap 0 and $m+1$ and consider the transposed digraph $\left(V, A^{\top}\right)$ where $A^{\top}$ comprises all arcs $(j, i)$ for $(i, j) \in A$. We use the resource $T^{\text {num }}$ as the monotone resource: only labels that fulfill the half-way condition $T^{\text {num }} \leq Q / 2$ are extended. If the routing costs are symmetric (i.e., $c_{i j}=c_{j i}$ for all $i, j \in V$ ), it becomes even simpler: all forward labels can be directly interpreted as backward labels (such an implicit bidirectional labeling was, e.g., described in the works of Bode and Irnich, 2012; Goeke et al., 2019; Gschwind et al., 2019).

A forward label $L_{f w}=\left(i, S_{f w}, T_{f w}\right)$ and a backward label $L_{b w}=\left(i, S_{b w}, T_{b w}\right)$ can be merged, i.e., the concatenation $P=\left(P_{f w}, i n v\left(P_{b w}\right)\right)$ of the two associated paths $P_{f w}=P\left(L_{f w}\right)$ and $P_{b w}=P\left(L_{b w}\right)$ is feasible if the following conditions hold:

$$
\begin{aligned}
T_{f w}^{n u m}+T_{b w}^{n u m} & \leq Q+1 \\
T_{f w}^{n g, k}+T_{b w}^{n g, k} & \leq 1 \quad \text { for all } k \in N_{i} \backslash\{i\}
\end{aligned}
$$

Condition (12a) reflects feasibility condition (10), whereas condition (12b) refers to the $n g$-path relaxation. The reduced cost of the merged path $P$ can be obtained as $c^{P}=T_{f w}^{c o s t}+T_{b w}^{c o s t}+f\left(S_{f w} \cup S_{b w}\right)$.

\subsubsection{Handling of Side Constraints}

We now describe the handling of side constraints, i.e., SVCs, incompatibilities between products, and tour duration/length constraints in the column-generation algorithm that we propose.

Single-Visit Constraints. For the route-based formulation, the SVCs can directly be incorporated in the master problem by adding the following set of constraints.

$$
\sum_{r \in \Omega} \sum_{j:(i, j) \in A} b_{i j}^{r} \lambda^{r} \leq 1 \quad i \in M
$$

with dual prices $\left(\pi_{i}\right)_{i \in M}$.

Moreover, by exploiting the SVCs, we can reduce the solution space of the subproblem by imposing that all products $K_{i}^{*}$ are purchased directly at the point when supplier $i \in M$ is visited because of Property 2 .

Property 2. If the triangle inequality holds for travel costs $\left(c_{i j}\right)$ then there exists an optimal solution to UMVTPP with SVCs in which at least $\max \left\{1,\left|K_{i}^{*}\right|\right\}$ products are purchased at every visited supplier $i \in M$.

Proof. See (Riera-Ledesma and Salazar-González, 2012).

To this end, we divide all products into unique and common products. Unique products $\bigcup_{i \in M} K_{i}^{*}$ have to be purchased and can be directly incorporated into the reduced cost of a partial path by using routing costs

$$
c_{i j}^{r e q}=c_{i j}+\frac{1}{2} \cdot\left(\sum_{k \in K_{i}^{*}} \bar{p}_{k i}+\sum_{k \in K_{j}^{*}} \bar{p}_{k j}\right)+\frac{1}{2} \cdot\left(\pi_{i}+\pi_{j}\right)
$$


in (9b) instead of $c_{i j}$ (we define $\pi_{0}=\pi_{m+1}=0$ for the depot vertices 0 and $m+1$ ). Note that the new reduced routing costs $c_{i j}^{r e q}$ can be negative. Moreover, for symmetric $c_{i j}$ the given definition of $c_{i j}^{r e q}$ implies that these are then also symmetric.

The labeling algorithm takes the unique and common products into account as follows: For all unique products $k \in K_{i}^{*}, i \in M$, we set $q_{k i}=0$ and $\bar{p}_{k i}=\infty$ to exclude unique products from being selected as common products, i.e., they do not occur in the sequence $O$ of a label.

To keep track of the number of unique products purchased, we introduce a new attribute $T^{\text {uniq }}$ for the labels. When propagating a label over an $\operatorname{arc}(i, j) \in A$, the new attribute is updated via $T_{j}^{u n i q}=$ $T_{i}^{\text {uniq }}+\left|K_{j}^{*}\right|$. The minimum number of collected common products is $T_{j}^{\text {num }}=T_{i}^{\text {num }}+1$ if $j \neq m+1$ and $K_{j}^{*}=\varnothing$, otherwise $T_{j}^{\text {num }}=T_{i}^{\text {num }}$ (this update rule replaces (9c)). The new label has the following additional feasibility conditions (in addition to $T_{j}^{\text {num }} \leq Q$, i.e., condition (10)): Not more than $Q$ unique products can be purchased, i.e., $T_{j}^{u n i q} \leq Q$, and the maximum number of common products purchased is now bounded by $T_{j}^{\text {num }} \leq Q-T_{j}^{u n i q}=Q-T_{i}^{\text {uniq }}-\left|K_{j}^{*}\right|$.

Regarding domination, a label $L_{1}$ dominates another label $L_{2}$, if in addition to (11a) and (11b) the condition $T_{1}^{\text {uniq }} \leq T_{2}^{\text {uniq }}$ holds.

For the bidirectional labeling, the monotone resource is redefined as $T^{\text {num }}+T^{\text {uniq }}$ with identical half-way point $Q / 2$ as before. Moreover, the merge conditions

$$
\begin{aligned}
& T_{f w}^{n u m}+T_{b w}^{n u m} \leq\left\{\begin{array}{l}
Q+1, \text { if }\left|K_{i}^{*}\right|=0 \\
Q, \text { otherwise }
\end{array}\right. \\
& T_{f w}^{u n i q}+T_{b w}^{u n i q} \leq Q+\left|K_{i}^{*}\right|
\end{aligned}
$$

have to be tested instead of (12a). Condition (14a) and (14b) refers respectively to the common and unique products. Note that, in the second constraint (14b), both the forward label and the backward label already take the unique products $K_{i}^{*}$ into account that are only available at the merge vertex $i$.

Incompatibilities between Products. Gendreau et al. (2016) deal with incompatibilities between products directly in the labeling algorithm. They introduce additional resources that guarantee that only compatible products are selected along the partial path.

We propose another approach to cope with incompatibilities: We enumerate all maximal subsets of products that are pairwise compatible. These are the maximal independent sets $I$ of the incompatibility graph $\left(K, E_{w}\right)$, where an edge $\left\{k, k^{\prime}\right\} \in E_{w}$ exists between products if and only if $\left(k, k^{\prime}\right) \in B$; see Section 1 . Let $\mathcal{I}=\left\{I_{1}, I_{2}, \ldots, I_{b}\right\}$ be the set of all maximum independent sets. The computation of the set $\mathcal{I}$ can be done with a straightforward enumeration algorithm.

The pricing subproblem can now be decomposed into $b$ subproblems, where in the subproblem to $I_{j}$ for $j \in\{1,2, \ldots, b\}$ only the products $I_{j} \subset K$ are available. For all suppliers, their product set $K_{i}$ is reduced to $K_{i} \cap I_{j}$. If the set is empty and the triangle inequality for routing costs holds, the supplier becomes obsolete. Note that in the presence of incompatibility constraints, the same supplier may be visited by multiple vehicles and it is therefore not possible to impose SVCs and distinguish between unique and common products.

On the positive side, the $b$ different column-generation subproblems are of the same structure as those discussed before. They can be solved with the labeling algorithm presented in Section 2.2.1. Due to the reduced product and supplier sets, one can expect that these subproblems can be solved faster than one subproblem with additional resources to cope with incompatibilities. On the downside, instead of one subproblem, there are $|\mathcal{I}|$ different subproblems. To avoid solving $|\mathcal{I}|$ subproblems in each iteration, partial pricing (Gamache et al., 1999) can be used to accelerate the solution process. We terminate the pricing when a certain number of negative reduced-cost columns is found. However, all $b$ subproblems must be solved in the final columngeneration iteration to prove optimality of the linear relaxation (per branch-and-bound node).

Maximum Route Duration Constraints. The integration of a maximum route duration constraint is simple. We add the attribute $T^{\text {length }}$ to the attributes vector $T$ of each label $L=(i, S, T)$. Initially the attribute is set to $T^{\text {length }}=0$. Extensions along an $\operatorname{arc}(i, j) \in A$ increase the attribute by $d_{i j}$, i.e., $T_{j}^{\text {length }}=T_{i}^{\text {length }}+d_{i j}$. The extension is feasible if $T_{j}^{\text {length }} \leq D^{\max }$. Dominance between two labels $L=\left(i, S_{1}, T_{1}\right)$ and $L=\left(i, S_{2}, T_{2}\right)$ has to additionally check $T_{1}^{\text {length }} \leq T_{2}^{\text {length }}$. The merge of a forward label $L_{f w}=\left(i, S_{f w}, T_{f w}\right)$ and $L_{b w}=$ $\left(i, S_{b w}, T_{b w}\right)$ must additionally test $T_{f w}^{\text {length }}+T_{b w}^{\text {length }} \leq D^{\max }$. 


\subsection{Valid Inequalities and Cutting Strategy}

For the UMVTPP with SVCs, the initial RMP that we use does not contain the SVCs (13). Instead, we add SVCs dynamically, i.e., when we find them violated.

In addition, four classes of valid inequalities are dynamically separated and added to the RMP in order to strengthen the linear relaxation. These inequalities require different properties of the problem that are summarized in Table 3 .

\begin{tabular}{lccc}
\hline & \multicolumn{3}{c}{ Prerequisite } \\
\cline { 2 - 4 } Valid inequalities & $\begin{array}{c}\text { Unitary } \\
\text { demand }\end{array}$ & $\begin{array}{c}\text { Single-visit } \\
\text { constraints (SVCs) }\end{array}$ & $\begin{array}{c}\text { Triangle Ineq. } \\
\text { for costs } c_{i j}\end{array}$ \\
\hline (Limited memory) subset-row inequalities, (lm)SRIs & & $\bullet$ \\
Rounded ring-capacity inequalities, RRCIs & $\bullet$ & \\
Ci-ring-capacity inequalities, CiRCIs & $\bullet$ & $\bullet$ \\
Purchase-visit inequalities, PVIs & $\bullet$ & $\bullet$ \\
\hline
\end{tabular}

Table 3: Prerequisites for the validity of inequalities

Subset-row inequalities (SRIs) were originally introduced by Jepsen et al. (2008) for the vehicle routing problem with time windows. This class of inequalities can only be used for UMVTPP variants with SVCs. In the UMVTPP, each SRI is defined for a subset $U \subset M$ of the suppliers. As proposed by Jepsen et al. (2008), we restrict ourselves to SRIs defined on three suppliers, i.e., $|U|=3$, because they can be separated by straightforward enumeration. The corresponding SRI is

$$
\sum_{r \in \Omega}\left\lfloor\frac{g_{r}^{U}}{2}\right\rfloor \lambda^{r} \leq 1
$$

where $g_{r}^{U}=\sum_{i \in U} a_{i r}$ is the number of times route $r \in \Omega$ visits suppliers in $U$.

SRIs comprise a family of non-robust cuts meaning that for each active SRI, i.e., with non-zero dual price, one binary attribute $T^{S R, U}$ must be added to the labels. The initial value of $T^{S R, U}$ is zero and it is flipped (from zero to one, and vice versa) every time a supplier $i \in U$ is visited. The (non-positive) dual price of the SRI is subtracted when the attribute flips from one to zero. A higher value $T_{1}^{S R, U}=1$ in a first label $L_{1}=\left(i, S_{1}, T_{1}\right)$ compared to $T_{2}^{S R, U}=0$ in a second label $L_{2}=\left(i, S_{2}, T_{1}\right)$ can be compensated by replacing the cost comparison (11a) by $T_{1}^{\text {cost }}+f\left(S_{1}\right)-\sum_{U: T_{1}^{S R, U}>T_{2}^{S R, U}} \alpha^{U} \leq T_{2}^{\text {cost }}+f\left(S_{1} \cup S_{2}\right)$, where $\alpha^{U} \leq 0$ is the dual price of the SRI related to the subset $U$. Even with this clever dominance rule of Jepsen et al. (2008), the presence of many SRIs for different subsets $U$ often drastically increases the practical difficulty of the pricing problem.

To alleviate these negative effects, Pecin et al. (2017) introduced limited memory SRIs (lmSRIs) that are a generalization of SRIs and whose impact on the difficulty of the pricing subproblem is typically reduced by using a $U$-specific memory to store a given subset $M_{U} \subseteq M$ of the suppliers. The role of the memory is very similar to the neighborhoods in the $n g$-path relaxation of Baldacci et al. (2011). The attribute $T^{S R, U}$ is reset to zero when a vertex $i$ outside the memory, i.e., $i \notin M_{U}$, is visited.

Rounded ring-capacity inequalities (RRCIs) and Ci-ring-capacity inequalities (CiRCIs) were introduced in the work (Baldacci et al., 2007) for the CmRSP. They are defined for an arbitrary subset $S \subset M$ of suppliers. Let $K(S)=\bigcup_{i \in S} K_{i}$ be the union of all products available at all suppliers in $S$. As for other capacity cuts, RRCIs and CiRCIs impose a lower bound on the number of times the subset $S$ must be exited:

$$
\sum_{r \in \Omega} \sum_{(i, j) \in \Gamma^{+}(S)} b_{i j}^{r} \lambda^{r}+\sum_{r \in \Omega} \frac{\sum_{i \notin S} \sum_{k \in K(S)} \delta_{k i}^{r}}{|K(S)|(\bmod Q)} \lambda^{r} \geq\left\lceil\frac{|K(S)|}{Q}\right\rceil
$$

and

$$
\sum_{r \in \Omega} \sum_{(i, j) \in \Gamma^{+}(S)} b_{i j}^{r} \lambda^{r} \geq\left\lceil\frac{\left|\left\{k \in K: M_{k} \subseteq S\right\}\right|}{Q}\right\rceil .
$$


Note that RRCIs result from some non-trivial inequalities that round fractions of integers. Hence they are difficult to interpret and we refer to (Baldacci et al., 2007) for the description and proof. In contrast, CiRCIs are simple to interpret: the left-hand side counts the number of times the subset $S$ is exited, while the nominator on the right-hand side counts the number of products that can only be purchased at suppliers inside $S$ so that the right-hand side term is the minimum number of vehicles needed to collect these products. For the separation of violated RRCIs and CiRCIs, we use the heuristic procedure suggested by Baldacci et al. (2007).

Finally, for UMVTPP with SVCs, in which the triangle inequality for the routing costs holds, we introduce a new class of valid inequalities that we denote as purchase-visit inequalities (PVIs). This class of inequalities exploits Property 2, i.e., that at each visited supplier $i \in M$ at least $\max \left\{1,\left|K_{i}^{*}\right|\right\}$ products must be purchased. The inequality for supplier $i \in M$ is then given by

$$
\sum_{r \in \Omega}\left(\max \left\{1,\left|K_{i}^{*}\right|\right\} \sum_{(i, j) \in \Gamma^{+}(i)} b_{i j}^{r}-\sum_{k \in K_{i}} \delta_{k i}^{r}\right) \lambda^{r} \leq 0 .
$$

For lmSRIs, RRCIs, CiRCIs, and PVIs, we use the following separation strategies. All inequalities are separated in hierarchical order, and the next class is only separated if we have found no violated inequalities of the previous class. The search for violated inequalities is limited to search-tree nodes of the overall branchand-bound tree up to a certain level $\max ^{\text {level }}$. For each round of separation, only the max ${ }^{\text {round }}$ most violated inequalities found are added before the RMP is re-optimized. The total number of separated inequalities per class is limited to $\max ^{\text {total }}$. Table 4 summarizes the values we used in our experiments.

\begin{tabular}{lcccc}
\hline & PVIs & CiRCIs & RRCIs & lmSRIs \\
\hline Order & 1 & 2 & 3 & 4 \\
Separated up to level max ${ }^{\text {level }}$ & $\infty$ & 10 & 10 & 1 \\
Maximum number $\max ^{\text {round }}$ per round & $|M|$ & 30 & 30 & 20 \\
Maximum number max ${ }^{\text {total }}$ in total & $|M|$ & 300 & 300 & 60 \\
\hline
\end{tabular}

Table 4: Parameters of the separation strategy for the four classes of valid inequalities

\subsection{Dynamic Neighborhood Extension}

Dynamic neighborhood extension (DNE) is a strategy to initialize and modify $n g$-neighborhoods $N_{i}$ depending on the solutions obtained at branch-and-bound nodes. DNE was first proposed by Roberti and Mingozzi (2014). The enlargement of neighborhoods can be interpreted as a tool to strengthen the linear relaxations. Note that a basic tradeoff exists between the strength of the lower bounds resulting from $n g$ neighborhoods $N_{i}$ of given size and the required computation time for the solution of the corresponding subproblems.

We implement DNE with the help of two integer parameters $s z_{\text {init }}^{n g}$ and $s z_{\text {max }}^{n g}$. Recall that cycles are the result of considering SVCs (and distinguishing between unique and common products) and adding cutting planes and branching constraints. As a consequence, negative reduced cost cycles at the root node certainly include some supplier $i$ at least twice that offers a unique product, i.e., $K_{i}^{*} \neq \varnothing$. Therefore, we add to the initial neighborhood of $j \in M$ the $s z_{\text {init }}^{n g}$ closest suppliers $i$ with $K_{i}^{*} \neq \varnothing$, so that $\left|N_{i}\right| \leq s z_{\text {init }}^{n g}$ holds for all $i \in M$.

These initial neighborhoods are systematically enlarged considering the solution of linear relaxations at each node of the search tree. If a fractional solution contains a route with a cycle $D=(i, \ldots, j, \ldots, i)$, we try to eliminate this cycle by adding $i$ to all neighborhoods of vertices $j$ in the cycle. Before actually adding supplier $i$, we check that all neighborhoods $N_{j}$ admit the addition, i.e., we check $\left|N_{j}\right|<s z_{\max }^{n g}$. Given the solution of the current RMP defined over $\bar{\Omega}$, let $\bar{\lambda}_{r}, r \in \bar{\Omega}$, be the value of the corresponding variable $\lambda_{r}$. Cycles in routes $r$ are considered in decreasing order of the values $\bar{\lambda}_{r}$. Our implementation uses $s z_{\text {init }}^{n g}=2$ and $s z_{\text {max }}^{n g}=8$.

\subsection{Branching}

Let $\bar{\lambda}_{r}$ for $r \in \bar{\Omega}$ and $\bar{f}$ be the values of the corresponding variables $\lambda_{r}$ and $f$ in the current solution of the RMP defined over $\bar{\Omega}$. If some of these values are fractional, branching is required. 


\begin{tabular}{cclllll}
\hline Stage & $\begin{array}{c}\text { SVCs } \\
\text { with w/o }\end{array}$ & Description & $\begin{array}{l}\text { Branching } \\
\text { on }\end{array}$ & $\begin{array}{l}\text { Variable } \\
\text { selection }\end{array}$ & Branches \\
\hline 1 & $\bullet$ & $\bullet$ & Number of vehicles & $\bar{f}$ & - & $\leq\lfloor\bar{f}\rfloor$ and $\geq\lceil\bar{f}\rceil$ \\
2 & $\bullet$ & $\bullet$ & Selection of a supplier & $\alpha_{k i}, \quad k \in K, i \in M_{k}$ & closest to 0.5 & $=0$ and $=1$ \\
3 & & $\bullet$ & Number of visits & $\gamma_{i}, \quad i \in M$ & $\gamma_{i}-\left\lfloor\gamma_{i}\right\rfloor$ closest to 0.5 & $\leq\left\lfloor\gamma_{i}\right\rfloor$ and $\geq\left\lceil\gamma_{i}\right\rceil$ \\
4 & $\bullet$ & $\bullet$ & Slack of CiRCI & $\beta_{S}, \quad S \subset M$ & $<1$, closest to $0.5 \quad \leq$ and $\geq 1$ \\
5 & $\bullet$ & $\bullet$ & Arc Flow & $\eta_{i j},(i, j) \in A$ & $\eta_{i j}-\left\lfloor\eta_{i j}\right\rfloor$ closest to $0.5 \quad \leq\left\lfloor\eta_{i j}\right\rfloor$ and $\geq\left\lceil\eta_{i j}\right\rceil$ \\
6 & $\bullet$ & $\bullet$ & Flow splitting & see (Feillet et al., 2005) for details, not necessary in our experiments \\
\hline
\end{tabular}

Table 5: Six-stage branching strategy for the UMVTPP

We apply a six-stage hierarchical branching scheme to ensure integer solutions of formulation (1). For the UMVTPP with SVCs, the third stage is obsolete. Table 5 provides an overview.

First, if the number of vehicles $\bar{f}$ in use is fractional, we create two branches enforcing either $f \leq\lfloor\bar{f}\rfloor$ or $f \geq\lceil\bar{f}\rceil$.

Second, we introduce a new branching rule that decides whether product $k \in K$ is purchased at supplier $i \in M$, or not (the use of this branching rule is new compared to Hoshino and De Souza, 2012; Gendreau et al., 2016). For all products $k \in K$ with $\left|M_{k}\right|>1$ and all suppliers $i \in M_{k}$, we consider fractional values $\alpha_{k i}$ defined as $\sum_{r \in \Omega} \delta_{k i} \bar{\lambda}_{r}$. We choose a pair $\left(k^{*}, i^{*}\right)$ with value $\alpha_{k^{*} i^{*}}$ closest to 0.5 and create two branches $\sum_{r \in \Omega} \delta_{k i} \lambda_{r}=0$ and $\sum_{r \in \Omega} \delta_{k i} \lambda_{r}=1$. Instead of adding constraints, we directly implement the branching constraints by manipulating the product set of the suppliers. In the first branch, product $k^{*}$ is removed from $K_{i^{*}}$. In the second branch, product $k^{*}$ is removed from $K_{i}$ for all other suppliers $i \in M_{k} \backslash\left\{i^{*}\right\}$; this ensures that product $k^{*}$ is purchased at supplier $i^{*}$. All route variables that do not comply with the branching decision are removed from the RMP.

Third, we branch on the number of times a supplier $i \in M$ is visited if this value is fractional. Accordingly, we define $\gamma_{i}$ as $\sum_{r \in \Omega} \sum_{(i, j) \in \Gamma^{+}(i)} b_{i j}^{r} \bar{\lambda}^{r}$. Among all suppliers $i \in M$ with fractional $\gamma_{i}$, we choose a supplier $i^{*}$ with $\gamma_{i^{*}}-\left\lfloor\gamma_{i^{*}}\right\rfloor$ closest to 0.5 and create two branches with either

$$
\sum_{r \in \Omega} \sum_{(i, j) \in \Gamma^{+}(i)} b_{i j}^{r} \lambda^{r} \leq\left\lfloor\gamma_{i^{*}}\right\rfloor \quad \text { or } \quad \sum_{r \in \Omega} \sum_{(i, j) \in \Gamma^{+}(i)} b_{i j}^{r} \lambda^{r} \geq\left\lceil\gamma_{i^{*}}\right\rceil
$$

Note that this branching rule is obsolete for UMVTPP with SVCs when the second-stage branching rules are applied before.

Fourth, as suggested by Baldacci et al. (2007), we branch on the slack in CiRCIs. Recall that for any subset $S \subset M$, the corresponding CiRCI ensures that the subset $S$ is exited sufficiently often. Defining $\beta_{S}$ as $\sum_{r \in \Omega} \sum_{(i, j) \in \Gamma^{+}(S)} b_{i j}^{r} \bar{\lambda}^{r}-\left[\frac{\left|\left\{k \in K: M_{k} \subseteq S\right\}\right|}{Q}\right]$, we consider only those subsets $S$ with a value $\beta_{S}$ strictly between 0 and 1. We use the heuristic proposed by Baldacci et al. (2007) to identify candidate sets $S$. Among all candidate sets, we choose a subset $S^{*}$ with a value $\beta_{S^{*}}$ closest to 0.5 and create two branches by adding the additional constraints that either the slack is zero or at least one. The constraints defining these branches can be written as

$$
\sum_{r \in \Omega} \sum_{(i, j) \in \Gamma^{+}\left(S^{*}\right)} b_{i j}^{r} \lambda^{r}-\left\lceil\frac{\left|\left\{k \in K: M_{k} \subseteq S^{*}\right\}\right|}{Q}\right\rceil=0 \quad \text { or } \quad \geq 1 .
$$

Fifth, we branch on the number of times an $\operatorname{arc}(i, j) \in A$ is traversed. Let $\eta_{i j}$ be defined by $\sum_{r \in \Omega} b_{i j}^{r} \bar{\lambda}^{r}$, i.e., the number of times $\operatorname{arc}(i, j) \in A$ is used in the current solution. Among all arcs with fractional value $\eta_{i j}$, we choose the $\operatorname{arc}\left(i^{*}, j^{*}\right)$ with $\eta_{i^{*} j^{*}}$ closest to 0.5 . We create two branches by forcing $\sum_{r \in \Omega} b_{i j}^{r} \lambda^{r} \leq\left\lfloor\eta_{i^{*} j^{*}}\right\rfloor$ or $\sum_{r \in \Omega} b_{i j}^{r} \lambda^{r} \geq\left\lceil\eta_{i^{*} j^{*}}\right\rceil$. This branching rule reduces to standard binary arc branching for the UMVTPP with SVCs.

Note that integer flows on arcs may not guarantee that the route variables are integer for UMVTPPs without SVCs. Therefore, the sixth stage applies the additional branching rule based on the flow-splitting method introduced by Feillet et al. (2005) and later used also by Gendreau et al. (2016). In our computational experiments, it was never necessary to apply this rule because fractional route variables could always be convex combined into feasible integer solutions (see, Desaulniers et al., 1998; Jans, 2010). We attribute this behavior to the use of the additional second-stage branching rule not used by Gendreau et al. (2016). We doubt however that the first five stages already guarantee, in general, that the branching is complete. 


\section{Computational Results}

In this section, we report the results of computational experiments that were conducted on different classes of UMVTPP instances (Section 3.1) using additional acceleration techniques based on partial pricing (Section 3.2). We compare our BPC algorithm with algorithms from the literature on three standard benchmark sets in Section 3.3.

We have implemented the BPC algorithm in $\mathrm{C}++$ and compiled into 64-bit single-thread code with MS Visual Studio 2015. The callable library of CPLEX 12.9.0 was used for (re-)optimizing the RMPs. All results were obtained using a standard PC with an Intel ${ }^{\circledR}$ Core $^{\mathrm{TM}}$ i7-5930K processor clocked at $3.5 \mathrm{GHz}$ and 64 GB RAM running Microsoft Windows 10 Education. In the following, all computation times are given in seconds.

\subsection{UMVTPP Instances}

We test our BPC algorithm on the CmRSP instances created by Baldacci et al. (2007) and Hoshino and De Souza (2012) as well as on the MVTPP-PIC instances of Gendreau et al. (2016). Several comments on these benchmarks sets and previous results presented in the literature are due:

1. The CmRSP instances of Baldacci et al. (2007) have been used in previous studies only in a restricted version, where a canonical ring-star property has been assumed. Hoshino and De Souza (2012, p. 2734f) already mention that Baldacci et al. assume canonical ring stars in which a Steiner point appears in a ring only if there exists a connection arc in the star linking some customer to it. Only if routing costs satisfy the triangle inequality, there always exists an optimal solution composed exclusively of canonical ring stars. Otherwise, all optimal solutions may have non-canonical ring stars. Since the triangle inequality is not fulfilled in this benchmark set, the solutions reported in (Baldacci et al., 2007) may not be optimal. Moreover, in order to speed up the convergence of the solution process, the authors provided their BC algorithm with initial feasible solutions (computed by applying at pre-processing time an initial standalone heuristic) whose values were, on average, less than $0.7 \%$ away from the optimal (dual bound) values. Finally, it should also be noted that capacities have been chosen large for some of these instances, which makes them somewhat harder for column-generation based algorithms but simpler for BC algorithms.

2. Hoshino and De Souza (2012) also forced the ring stars to be canonical to be in conformity with Baldacci et al.. However, they adopt the instances of Baldacci et al., such that the triangle inequality is satisfied in their new benchmark set. As their BC results are inferior to their BPC results, we do not include the BC results into our comparison in Section 3.3. Additionally, in order to further assess their BPC algorithm, the authors run a subset of experiments considering the instances proposed by Baldacci et al.. Their aggregated results show that the BPC algorithm solves less instances to optimality compared to the BC results of Baldacci et al..

3. Riera-Ledesma and Salazar-González $(2012,2013)$ extended the original benchmark set comprising the two classes A and B of Baldacci et al. by three new classes C, D, and E. Unfortunately, these instances are not obtainable anymore (Riera-Ledesma, 2019).

4. Instances of the MVTPP-PIC benchmark set of Gendreau et al. (2016) do not fulfill the triangle inequality for routing costs. Therefore, Property 1 does not hold true, i.e., visits to suppliers without actually purchasing any product can be beneficial. In addition, their BP algorithm has been prematurely terminated whenever the optimality gap has been fallen below 0.2 percent. Hence, some non-optimal solutions are reported in their results.

Finally, we would like to mention that the instances of Bianchessi et al. (2014) were created and tested only for uncapacitated variants of the MVTPP. In this case, the purchasing decisions can be transferred to the master-problem level of a column-generation approach (as done by the authors). This makes these instances irrelevant for any approach that explicitly considers capacities on the subproblem level. We therefore disregard these instances.

\subsection{Partial Pricing}

To accelerate the solution process, we use partial pricing with reduced networks. A reduced network is defined by a value $\alpha \in \mathbb{N}$ that limits the number of ingoing and outgoing arcs per vertex. Arcs are chosen according to the lowest reduced cost in the current pricing iteration.

Additionally, we use two heuristic dominance rule. In the first rule ('rule 1'), condition (11a) is replaced by

$$
T_{1}^{\text {cost }}+f\left(S_{1}\right) \leq T_{2}^{\text {cost }}+f\left(S_{2}\right)
$$


and in the second rule ('rule 2'), condition (11a) is replaced by

$$
T_{1}^{\text {cost }}+f\left(S_{1}\right) \leq T_{2}^{\text {cost }}+\beta f\left(S_{1} \cup S_{2}\right)
$$

with some value $\beta$ between 0 and 1 . In our computational experiments, we used six heuristic pricers. Their configuration is summarized in Table 6 .

\begin{tabular}{lrrr}
\hline Level & $\begin{array}{r}\text { Number } \alpha \\
\text { of arcs }\end{array}$ & $\begin{array}{r}\text { Dominance } \\
\text { rule }\end{array}$ & $\begin{array}{r}\text { Factor } \beta \\
\text { in rule } 2\end{array}$ \\
\hline 1 & 20 & rule 1 & - \\
2 & $\infty$ & rule 1 & - \\
3 & 30 & rule 2 & 0.85 \\
4 & 30 & rule 2 & 0.95 \\
5 & 10 & exact & 1 \\
6 & 20 & exact & 1 \\
\hline
\end{tabular}

Table 6: Configuration of heuristic pricers

Due to the restricted and hence smaller set of products and suppliers per pricing subproblem in the MVTPP-PIC (see Section 2.2.2), we skip heuristic pricers at levels 1, 3, and 4.

\subsection{Results for the CmRSP and the MVTPP-PIC Instances}

In this section, we compare our BPC algorithm on instances for the CmRSP and the MVTPP-PIC with the state-of-the-art algorithms from the literature. Tables 7-9 present aggregated results for the instances of Baldacci et al. (2007), Hoshino and De Souza (2012), and Gendreau et al. (2016). Detailed instance-wise results can be found in the Appendix. We set the same time limit as in the literature: 7200 seconds for the instances of Baldacci et al. (2007) and Gendreau et al. (2016) as well as 1800 seconds for the instances of Hoshino and De Souza (2012). The tables contain for each instance class, the number of instances (\#), the number of instances solved to optimality (\#Opt), the average solution time in seconds, and the number of branch-and-bound nodes solved (\#B\&B).

\begin{tabular}{|c|c|c|c|c|c|c|c|c|}
\hline \multirow[b]{2}{*}{ Instance } & & \multirow[b]{2}{*}{$\#$} & \multicolumn{3}{|c|}{ Baldacci et al. } & \multicolumn{3}{|c|}{ Our algorithm } \\
\hline & & & $\# \mathrm{Opt}$ & Time & $\# \mathrm{~B} \& \mathrm{~B}$ & $\# \mathrm{Opt}$ & Time & $\# \mathrm{~B} \& \mathrm{~B}$ \\
\hline \multicolumn{9}{|c|}{ grouped by class } \\
\hline & A 25 & 9 & 9 & 0.7 & 1.1 & 9 & 0.3 & 4.9 \\
\hline & A50 & 12 & 12 & 570.6 & 323.9 & 10 & 1659.2 & 54.8 \\
\hline & A75 & 12 & 8 & 2942.1 & 590.8 & 4 & 4950.4 & 252.7 \\
\hline & A100 & 12 & 6 & 4355.4 & 434.0 & 3 & 5424.9 & 9.3 \\
\hline & B25 & 9 & 9 & 0.4 & 1.0 & 9 & 0.3 & 5.7 \\
\hline & B50 & 12 & 12 & 613.1 & 230.3 & 12 & 655.8 & 43.5 \\
\hline & B75 & 12 & 4 & 4976.5 & 597.1 & 3 & 5418.8 & 711.5 \\
\hline & B100 & 12 & 3 & 5483.5 & 406.3 & 3 & 5458.8 & 31.1 \\
\hline \multicolumn{9}{|c|}{ grouped by vehicle capacity } \\
\hline & $3-6$ & 26 & 26 & 67.9 & 14.3 & 26 & 3.3 & 10.7 \\
\hline & $7-10$ & 20 & 19 & 415.9 & 54.8 & 19 & 511.7 & 597.7 \\
\hline & $11-14$ & 18 & 11 & 3866.2 & 581.3 & 7 & 5027.9 & 57.4 \\
\hline & $\geq 15$ & 26 & 7 & 5678.0 & 733.8 & 1 & 7000.0 & 2.4 \\
\hline Total & & 90 & 63 & 2525.6 & 344.5 & 53 & 3142.4 & 148.1 \\
\hline
\end{tabular}

Table 7: Comparison with the branch-and-cut (BC) algorithm of Baldacci et al. (2007)

The first part of Table 7 contains aggregated results in which the instances are grouped by class (A or B) and number of suppliers (25 to 100). Afterwards, in the second part of the table, we regroup the instances by vehicle capacity. 
In the first part, we can see that our algorithm performs worse on the A instances, while it performs nearly equally on the B instances. From the second part we can see that both algorithms operate similarly on instances with a vehicle capacity up to 10 . The BC algorithm of Baldacci et al. (2007) is slightly better on instances with vehicle capacity between 11 and 14. However, we can provide two new optimal solutions for that group. As expected, our algorithm performs poorly for instances that have a vehicle capacity larger than 15. All in all, we can solve 10 instances less and our solution time increases on average.

\begin{tabular}{|c|c|c|c|c|c|c|c|}
\hline \multirow[b]{2}{*}{ Instance class } & \multirow[b]{2}{*}{$\#$} & \multicolumn{3}{|c|}{ Hoshino and De Souza } & \multicolumn{3}{|c|}{ Our algorithm } \\
\hline & & $\# \mathrm{Opt}$ & Time & $\# B \& B$ & \#Opt & Time & $\# B \& B$ \\
\hline Small A & 33 & 32 & 91.8 & 127.2 & 32 & 93.7 & 16.8 \\
\hline B & 33 & 32 & 110.4 & 165.8 & 32 & 152.5 & 40.7 \\
\hline C & 33 & 29 & 289.7 & 190.5 & 27 & 331.2 & 13.1 \\
\hline Large A & 45 & 18 & 1162.5 & 483.3 & 18 & 1118.8 & 36.7 \\
\hline B & 45 & 14 & 1269.1 & 828.3 & 16 & 1234.2 & 142.9 \\
\hline $\mathrm{C}$ & 45 & 10 & 1464.4 & 384.7 & 15 & 1234.2 & 53.7 \\
\hline Total & 234 & 135 & 818.6 & 394.4 & 140 & 694.1 & 50.6 \\
\hline
\end{tabular}

Table 8: Comparison with the branch-price-and-cut (BPC) algorithm of Hoshino and De Souza (2012)

Compared with the BPC algorithm of Hoshino and De Souza (2012), our algorithm performs slightly better on average. For the small instance class, both algorithms work similarly. The two instances of class small C that we cannot solve (solved by the BPC of Hoshino and De Souza (2012)) have a large vehicle capacity of 14 . For the large instance classes, our algorithm performs better, in particular for the large instances of class B and C. In total, we can solve five additional instances and the solution time decreases slightly on average. It is notable that we need significantly less branch-and-bound nodes to solve the instances to optimality. Moreover, the instance-wise results show that we can provide ten optimal solutions for previously unsolved instances.

\begin{tabular}{|c|c|c|c|c|c|c|c|c|}
\hline \multirow[b]{2}{*}{ Instance size $\mid M$} & \multirow[b]{2}{*}{+1} & \multirow[b]{2}{*}{$\#$} & \multicolumn{3}{|c|}{ Gendreau et al. } & \multicolumn{3}{|c|}{ Our algorithm } \\
\hline & & & $\# \mathrm{Opt}$ & Time & \#B\&B & $\# \mathrm{Opt}$ & Time & \#B\&B \\
\hline & 20 & 24 & 24 & 869.3 & 87.3 & 24 & 31.1 & 24.8 \\
\hline & 35 & 24 & 21 & 1906.9 & 8.5 & 24 & 93.0 & 20.6 \\
\hline & 50 & 24 & 13 & 4054.0 & 5.6 & 23 & 881.6 & 144.1 \\
\hline Total & & 72 & 58 & 2276.7 & 33.8 & 71 & 335.2 & 63.2 \\
\hline
\end{tabular}

Table 9: Comparison with the BPC algorithm of Gendreau et al. (2016).

Compared to the BPC algorithm of Gendreau et al. (2016), our BPC algorithm performs much better. We can solve all but one instance to optimality, thereby providing 19 new optimal solutions where six of them are resulting from the preliminary stop criterion in the BPC of Gendreau et al.. Moreover, our algorithm is on average more than six times faster, although the detailed results show (entry \#Sub of Table 18 in the Online Appendix) that there are up to 286 different pricing subproblems per instance.

\section{Conclusions}

In this work, we have presented a new branch-price-and-cut (BPC) algorithm for the solution of different variants of the capacitated multiple vehicle traveling purchaser problem (MVTPP) with unitary demand. The main novelty is the way in which the column-generation subproblems are solved by a dynamic-programming labeling algorithm: The precise purchasing decisions are postponed until the route has been completely defined. The decisive point here is the definition of an effective dominance rule. The novel dominance rule 
uses some deep-seated properties of the optimal reduced purchasing cost function, which we have proved being supermodular.

Additional components of the BPC algorithm that contributed to the overall effectiveness of the approach are implicit bidirectional and partial pricing, the incorporation of additional cutting planes, the dynamic extension of the $n g$-route neighborhoods, and new branching rules. For the UMVTPP with incompatibilities among products, the replacement of incompatibility constraints by multiple pricing subproblems has produced a BPC approach that outperforms former methods by one order of magnitude regarding computation times. In general, the computational results indicate that the new subproblem solution strategies are beneficial, especially for instances in which many products are available at a supplier and routes are relatively short.

\section{Acknowledgement}

This research was funded by the Deutsche Forschungsgemeinschaft (DFG) under grants no. IR 122/5-2 and IR 122/9-2 and by Regione Lombardia, grant agreement n. E97F17000000009, Project AD-COM.

\section{References}

Baldacci, R., Dell'Amico, M., and Salazar González, J. (2007). The capacitated m-ring-star problem. Operations Research, $\mathbf{5 5}(6), 1147-1162$.

Baldacci, R., Mingozzi, A., and Roberti, R. (2011). New route relaxation and pricing strategies for the vehicle routing problem. Operations Research, 59(5), 1269-1283.

Bianchessi, N. and Irnich, S. (2019). Branch-and-cut for the split delivery vehicle routing problem with time windows. Transportation Science, $\mathbf{5 3}(2), 442-462$.

Bianchessi, N., Mansini, R., and Speranza, M. (2014). The distance constrained multiple vehicle traveling purchaser problem. European Journal of Operational Research, 235(1), 73-87.

Bode, C. and Irnich, S. (2012). Cut-first branch-and-price-second for the capacitated arc-routing problem. Operations Research, 60(5), 1167-1182.

Choi, M. J. and Lee, S. H. (2010). The multiple traveling purchaser problem. In The 40th International Conference on Computers Indutrial Engineering, pages 1-5.

Christofides, N., Mingozzi, A., and Toth, P. (1981). Exact algorithms for the vehicle routing problem, based on spanning tree and shortest path relaxations. Mathematical Programming, 20(1), 255-282.

Desaulniers, G., Desrosiers, J., Ioachim, I., Solomon, M., Soumis, F., and Villeneuve, D. (1998). A unified framework for deterministic time constrained vehicle routing and crew scheduling problems. In T. G. Crainic and G. Laporte, editors, Fleet Management and Logistics, pages 57-93. Kluwer, Boston.

Desaulniers, G., Desrosiers, J., and Solomon, M., editors (2005). Column Generation. Springer, New York.

Feillet, D., Dejax, P., and Gendreau, M. (2005). The profitable arc tour problem: Solution with a branch-and-price algorithm. Transportation Science, 39(4), 539-552.

Gamache, M., Soumis, F., and Marquis, G. (1999). A column generation approach for large-scale aircrew rostering problems. Operations Research, 47(2), 247-263.

Gendreau, M., Manerba, D., and Mansini, R. (2016). The multi-vehicle traveling purchaser problem with pairwise incompatibility constraints and unitary demands: A branch-and-price approach. European Journal of Operational Research, 248(1), $59-71$.

Goeke, D., Gschwind, T., and Schneider, M. (2019). Upper and lower bounds for the vehicle-routing problem with private fleet and common carrier. Discrete Applied Mathematics, 264, 43-61.

Gschwind, T., Bianchessi, N., and Irnich, S. (2019). Stabilized branch-price-and-cut for the commodity-constrained split delivery vehicle routing problem. European Journal of Operational Research, 278(1), 91-104.

Hoshino, E. A. and De Souza, C. C. (2012). A branch-and-cut-and-price approach for the capacitated $m$-ring-star problem. Discrete Applied Mathematics, 160(18), 2728-2741.

Irnich, S. and Desaulniers, G. (2005). Shortest path problems with resource constraints. In G. Desaulniers, J. Desrosiers, and M. Solomon, editors, Column Generation, pages 33-65. Springer, New York.

Jans, R. (2010). Classification of Dantzig-Wolfe reformulations for binary mixed integer programming problems. European Journal of Operational Research, 204(2), 251-254.

Jepsen, M., Petersen, B., Spoorendonk, S., and Pisinger, D. (2008). Subset-row inequalities applied to the vehicle-routing problem with time windows. Operations Research, 56(2), 497-511.

Manerba, D. and Mansini, R. (2015). A branch-and-cut algorithm for the multi-vehicle traveling purchaser problem with pairwise incompatibility constraints. Networks, 65(2), 139-154.

Manerba, D. and Mansini, R. (2016). The nurse routing problem with workload constraints and incompatible services. IFACPapersOnLine, 49(12), 1192-1197. 8th IFAC Conference on Manufacturing Modelling, Management and Control MIM 2016.

Manerba, D., Mansini, R., and Riera-Ledesma, J. (2017). The traveling purchaser problem and its variants. European Journal of Operational Research, 259(1), 1-18.

Miller, C. E., Tucker, A. W., and Zemlin, R. A. (1960). Integer programming formulations and traveling salesman problem. Journal of the ACM, 7, 326-329.

Pecin, D., Pessoa, A., Poggi, M., and Uchoa, E. (2017). Improved branch-cut-and-price for capacitated vehicle routing. Mathematical Programming Computation, 9(1), 61-100.

Riera-Ledesma, J. (2019). Personal communication. 
Riera-Ledesma, J. and Salazar-González, J.-J. (2012). Solving school bus routing using the multiple vehicle traveling purchaser problem: A branch-and-cut approach. Computers \& Operations Research, 39(2), 391-404.

Riera-Ledesma, J. and Salazar-González, J. J. (2013). A column generation approach for a school bus routing problem with resource constraints. Computers $\&$ Operations Research, 40(2), 566-583.

Righini, G. and Salani, M. (2006). Symmetry helps: Bounded bi-directional dynamic programming for the elementary shortest path problem with resource constraints. Discrete Optimization, 3(3), 255-273.

Roberti, R. and Mingozzi, A. (2014). Dynamic ng-path relaxation for the delivery man problem. Transportation Science, 48(3), 413-424

Schrijver, A. (2003). Combinatorial Optimization. Springer, Berlin.

Tilk, C., Rothenbächer, A.-K., Gschwind, T., and Irnich, S. (2017). Asymmetry matters: Dynamic half-way points in bidirectional labeling for solving shortest path problems with resource constraints faster. European Journal of Operational Research, 261(2), 530-539. 


\section{Online Appendix}

Tables 10-18 present detailed computational results for all tested instances. The first column indicates the name of the instances, the second gives the number of suppliers and the third the number of products. Columns $L B$ und $U B$ contain the lower and upper bounds obtained at the end of the optimization. Columns Time LP and Time IP give the time the BPC algorithm takes for the solution of the root node and the complete IP, respectively. Column \#B\&B contains the number of branch-and-bound nodes solved, column \#ExtNG the number of nodes at which the ng-neighborhood was extended, and column \#Cuts the number of added cutting planes. Finally, for the instances with incompatibilities, column $f r$ denotes the percentage of products that are free, i.e., the percentage of products that have no incompatibility restrictions, and column \#Sub shows the number of subproblems for that instance.

\begin{tabular}{|c|c|c|c|c|c|c|c|c|c|}
\hline Instance & $|M|$ & $|K|$ & $U B$ & $L B$ & Time LP & Time IP & \#B\&B & \#ExtNG & \#Cuts \\
\hline eil26.tsp.3.12.5.A.BDS & 25 & 12 & 242 & 242 & 0.1 & 0.1 & 2 & 1 & 8 \\
\hline eil26.tsp.4.12.4.A.BDS & 25 & 12 & 261 & 261 & 0.1 & 0.1 & 2 & 1 & 7 \\
\hline eil26.tsp.5.12.3.A.BDS & 25 & 12 & 292 & 292 & 0.1 & 0.1 & 2 & 1 & 12 \\
\hline eil26.tsp.3.18.7.A.BDS & 25 & 18 & 301 & 301 & 0.1 & 0.8 & 14 & 7 & 57 \\
\hline eil26.tsp.4.18.5.A.BDS & 25 & 18 & 339 & 339 & 0.1 & 0.1 & 2 & 1 & 10 \\
\hline eil26.tsp.5.18.4.A.BDS & 25 & 18 & 375 & 375 & 0.1 & 0.1 & 2 & 1 & 17 \\
\hline eil26.tsp.3.25.10.A.BDS & 25 & 25 & 325 & 325 & 0.2 & 0.8 & 11 & 8 & 38 \\
\hline eil26.tsp.4.25.7.A.BDS & 25 & 25 & 362 & 362 & 0.1 & 0.4 & 7 & 5 & 26 \\
\hline eil26.tsp.5.25.6.A.BDS & 25 & 25 & 382 & 382 & 0.1 & 0.1 & 2 & 1 & 18 \\
\hline eil51.tsp.3.12.5.A.BDS & 50 & 12 & 242 & 242 & 0.1 & 0.1 & 2 & 1 & 8 \\
\hline eil51.tsp.4.12.4.A.BDS & 50 & 12 & 261 & 261 & 0.1 & 0.1 & 2 & 1 & 7 \\
\hline eil51.tsp.5.12.3.A.BDS & 50 & 12 & 286 & 286 & 0.1 & 0.1 & 2 & 1 & 8 \\
\hline eil51.tsp.3.25.10.A.BDS & 50 & 25 & 322 & 322 & 0.5 & 2.0 & 6 & 4 & 38 \\
\hline eil51.tsp.4.25.7.A.BDS & 50 & 25 & 360 & 360 & 0.3 & 1.3 & 10 & 5 & 53 \\
\hline eil51.tsp.5.25.6.A.BDS & 50 & 25 & 379 & 379 & 0.2 & 0.3 & 2 & 1 & 17 \\
\hline eil51.tsp.3.37.14.A.BDS & 50 & 37 & 373 & 373 & 7.3 & 491.7 & 40 & 19 & 71 \\
\hline eil51.tsp.4.37.11.A.BDS & 50 & 37 & 405 & 405 & 1.8 & 1395.2 & 338 & 130 & 112 \\
\hline eil51.tsp.5.37.9.A.BDS & 50 & 37 & 432 & 432 & 0.6 & 25.0 & 26 & 13 & 70 \\
\hline eil51.tsp.3.50.19.A.BDS & 50 & 50 & - & 455 & 199.6 & 7200.0 & 33 & 18 & 119 \\
\hline eil51.tsp.4.50.14.A.BDS & 50 & 50 & 490 & 490 & 39.1 & 3595.3 & 40 & 24 & 97 \\
\hline eil51.tsp.5.50.12.A.BDS & 50 & 50 & - & 518 & 14.1 & 7200.0 & 156 & 72 & 93 \\
\hline eil76.tsp.3.18.7.A.BDS & 75 & 18 & 330 & 330 & 0.4 & 1690.1 & 2947 & 227 & 135 \\
\hline eil76.tsp.4.18.5.A.BDS & 75 & 18 & 385 & 385 & 0.2 & 4.7 & 25 & 5 & 62 \\
\hline eil76.tsp.5.18.4.A.BDS & 75 & 18 & 448 & 448 & 0.2 & 4.9 & 28 & 9 & 68 \\
\hline eil76.tsp.3.37.14.A.BDS & 75 & 37 & - & 394 & 2442.0 & 7200.0 & 3 & 2 & 27 \\
\hline eil76.tsp.4.37.11.A.BDS & 75 & 37 & - & 440 & 178.0 & 7200.0 & 26 & 10 & 160 \\
\hline eil76.tsp.5.37.9.A.BDS & 75 & 37 & 479 & 479 & 43.9 & 105.2 & 3 & 0 & 4 \\
\hline eil76.tsp.3.56.21.A.BDS & 75 & 56 & - & - & 7200.0 & 7200.0 & 0 & 0 & 0 \\
\hline eil76.tsp.4.56.16.A.BDS & 75 & 56 & - & - & 7200.0 & 7200.0 & 0 & 0 & 0 \\
\hline eil76.tsp.5.56.13.A.BDS & 75 & 56 & - & - & 7200.0 & 7200.0 & 0 & 0 & 0 \\
\hline eil76.tsp.3.75.28.A.BDS & 75 & 75 & - & - & 7200.0 & 7200.0 & 0 & 0 & 0 \\
\hline eil76.tsp.4.75.21.A.BDS & 75 & 75 & - & - & 7200.0 & 7200.0 & 0 & 0 & 0 \\
\hline eil76.tsp.5.75.17.A.BDS & 75 & 75 & - & - & 7200.0 & 7200.0 & 0 & 0 & 0 \\
\hline eil101.tsp.3.25.10.A.BDS & 100 & 25 & 363 & 363 & 7.8 & 182.1 & 17 & 11 & 56 \\
\hline eil101.tsp.4.25.7.A.BDS & 100 & 25 & 415 & 415 & 1.7 & 89.9 & 60 & 27 & 159 \\
\hline eil101.tsp.5.25.6.A.BDS & 100 & 25 & 448 & 448 & 1.1 & 27.5 & 28 & 11 & 132 \\
\hline eil101.tsp.3.50.19.A.BDS & 100 & 50 & - & - & 7200.0 & 7200.0 & 0 & 0 & 0 \\
\hline eil101.tsp.4.50.14.A.BDS & 100 & 50 & - & - & 7200.0 & 7200.0 & 0 & 0 & 0 \\
\hline eil101.tsp.5.50.12.A.BDS & 100 & 50 & - & 551 & 1540.7 & 7200.0 & 6 & 3 & 35 \\
\hline eil101.tsp.3.75.28.A.BDS & 100 & 75 & - & - & 7200.0 & 7200.0 & 0 & 0 & 0 \\
\hline eil101.tsp.4.75.21.A.BDS & 100 & 75 & - & - & 7200.0 & 7200.0 & 0 & 0 & 0 \\
\hline eil101.tsp.5.75.17.A.BDS & 100 & 75 & - & - & 7200.0 & 7200.0 & 0 & 0 & 0 \\
\hline eil101.tsp.3.100.38.A.BDS & 100 & 100 & - & - & 7200.0 & 7200.0 & 0 & 0 & 0 \\
\hline eil101.tsp.4.100.28.A.BDS & 100 & 100 & - & - & 7200.0 & 7200.0 & 0 & 0 & 0 \\
\hline eil101.tsp.5.100.23.A.BDS & 100 & 100 & - & - & 7200.0 & 7200.0 & 0 & 0 & 0 \\
\hline
\end{tabular}

Table 10: Detailed Results for the instance class A of Baldacci et al. (2007) 


\begin{tabular}{|c|c|c|c|c|c|c|c|c|c|}
\hline Instance & $|M|$ & $|K|$ & $U B$ & $L B$ & Time LP & Time IP & \#B\&B & \#ExtNG & \#Cuts \\
\hline eil26.tsp.3.12.5.B.BDS & 25 & 12 & 1684 & 1684 & 0.1 & 0.1 & 4 & 3 & 8 \\
\hline eil26.tsp.4.12.4.B.BDS & 25 & 12 & 1827 & 1827 & 0.1 & 0.1 & 3 & 1 & 9 \\
\hline eil26.tsp.5.12.3.B.BDS & 25 & 12 & 2041 & 2041 & 0.1 & 0.0 & 2 & 1 & 12 \\
\hline eil26.tsp.3.18.7.B.BDS & 25 & 18 & 2104 & 2104 & 0.1 & 1.0 & 22 & 11 & 74 \\
\hline eil26.tsp.4.18.5.B.BDS & 25 & 18 & 2370 & 2370 & 0.1 & 0.1 & 3 & 2 & 14 \\
\hline eil26.tsp.5.18.4.B.BDS & 25 & 18 & 2615 & 2615 & 0.1 & 0.1 & 2 & 1 & 16 \\
\hline eil26.tsp.3.25.10.B.BDS & 25 & 25 & 2251 & 2251 & 0.1 & 0.5 & 5 & 3 & 30 \\
\hline eil26.tsp.4.25.7.B.BDS & 25 & 25 & 2510 & 2510 & 0.1 & 0.4 & 7 & 4 & 42 \\
\hline eil26.tsp.5.25.6.B.BDS & 25 & 25 & 2674 & 2674 & 0.1 & 0.2 & 3 & 2 & 23 \\
\hline eil51.tsp.3.12.5.B.BDS & 50 & 12 & 1681 & 1681 & 0.1 & 0.4 & 8 & 4 & 29 \\
\hline eil51.tsp.4.12.4.B.BDS & 50 & 12 & 1821 & 1821 & 0.1 & 0.2 & 7 & 4 & 18 \\
\hline eil51.tsp.5.12.3.B.BDS & 50 & 12 & 1972 & 1972 & 0.1 & 0.1 & 3 & 2 & 8 \\
\hline eil51.tsp.3.25.10.B.BDS & 50 & 25 & 2176 & 2176 & 0.6 & 0.9 & 2 & 1 & 21 \\
\hline eil51.tsp.4.25.7.B.BDS & 50 & 25 & 2470 & 2470 & 0.2 & 5.6 & 25 & 13 & 112 \\
\hline eil51.tsp.5.25.6.B.BDS & 50 & 25 & 2579 & 2579 & 0.2 & 0.4 & 2 & 1 & 25 \\
\hline eil51.tsp.3.37.14.B.BDS & 50 & 37 & 2490 & 2490 & 6.3 & 117.8 & 21 & 7 & 85 \\
\hline eil51.tsp.4.37.11.B.BDS & 50 & 37 & 2721 & 2721 & 1.2 & 978.5 & 304 & 97 & 103 \\
\hline eil51.tsp.5.37.9.B.BDS & 50 & 37 & 2908 & 2908 & 0.6 & 41.6 & 50 & 23 & 86 \\
\hline eil51.tsp.3.50.19.B.BDS & 50 & 50 & 3015 & 3015 & 104.3 & 2000.2 & 29 & 13 & 84 \\
\hline eil51.tsp.4.50.14.B.BDS & 50 & 50 & 3260 & 3260 & 18.0 & 4709.0 & 68 & 28 & 94 \\
\hline eil51.tsp.5.50.12.B.BDS & 50 & 50 & 3401 & 3401 & 6.7 & 14.7 & 3 & 2 & 17 \\
\hline eil76.tsp.3.18.7.B.BDS & 75 & 18 & 2259 & 2248 & 0.6 & 7200.0 & 8401 & 685 & 156 \\
\hline eil76.tsp.4.18.5.B.BDS & 75 & 18 & 2620 & 2620 & 0.3 & 2.8 & 18 & 5 & 50 \\
\hline eil76.tsp.5.18.4.B.BDS & 75 & 18 & 3059 & 3059 & 0.2 & 11.5 & 90 & 12 & 115 \\
\hline eil76.tsp.3.37.14.B.BDS & 75 & 37 & - & 2658 & 4803.2 & 7200.0 & 1 & 1 & 18 \\
\hline eil76.tsp.4.37.11.B.BDS & 75 & 37 & - & 2999 & 335.1 & 7200.0 & 21 & 7 & 113 \\
\hline eil76.tsp.5.37.9.B.BDS & 75 & 37 & 3284 & 3284 & 38.1 & 210.7 & 6 & 3 & 28 \\
\hline eil76.tsp.3.56.21.B.BDS & 75 & 56 & - & - & 7200.0 & 7200.0 & 0 & 0 & 0 \\
\hline eil76.tsp.4.56.16.B.BDS & 75 & 56 & - & - & 7200.0 & 7200.0 & 0 & 0 & 0 \\
\hline eil76.tsp.5.56.13.B.BDS & 75 & 56 & - & 3580 & 7034.8 & 7200.0 & 1 & 1 & 9 \\
\hline eil76.tsp.3.75.28.B.BDS & 75 & 75 & - & - & 7200.0 & 7200.0 & 0 & 0 & 0 \\
\hline eil76.tsp.4.75.21.B.BDS & 75 & 75 & - & - & 7200.0 & 7200.0 & 0 & 0 & 0 \\
\hline eil76.tsp.5.75.17.B.BDS & 75 & 75 & - & - & 7200.0 & 7200.0 & 0 & 0 & 0 \\
\hline eil101.tsp.3.25.10.B.BDS & 100 & 25 & 2434 & 2434 & 8.5 & 329.1 & 45 & 12 & 88 \\
\hline eil101.tsp.4.25.7.B.BDS & 100 & 25 & 2782 & 2782 & 1.8 & 345.7 & 289 & 67 & 141 \\
\hline eil101.tsp.5.25.6.B.BDS & 100 & 25 & 3009 & 3009 & 1.2 & 30.7 & 33 & 11 & 121 \\
\hline eil101.tsp.3.50.19.B.BDS & 100 & 50 & - & - & 7200.0 & 7200.0 & 0 & 0 & 0 \\
\hline eil101.tsp.4.50.14.B.BDS & 100 & 50 & - & - & 7200.0 & 7200.0 & 0 & 0 & 0 \\
\hline eil101.tsp.5.50.12.B.BDS & 100 & 50 & - & 3731 & 1017.3 & 7200.0 & 6 & 5 & 59 \\
\hline eil101.tsp.3.75.28.B.BDS & 100 & 75 & - & - & 7200.0 & 7200.0 & 0 & 0 & 0 \\
\hline eil101.tsp.4.75.21.B.BDS & 100 & 75 & - & - & 7200.0 & 7200.0 & 0 & 0 & 0 \\
\hline eil101.tsp.5.75.17.B.BDS & 100 & 75 & - & - & 7200.0 & 7200.0 & 0 & 0 & 0 \\
\hline eil101.tsp.3.100.38.B.BDS & 100 & 100 & - & - & 7200.0 & 7200.0 & 0 & 0 & 0 \\
\hline eil101.tsp.4.100.28.B.BDS & 100 & 100 & - & - & 7200.0 & 7200.0 & 0 & 0 & 0 \\
\hline eil101.tsp.5.100.23.B.BDS & 100 & 100 & - & - & 7200.0 & 7200.0 & 0 & 0 & 0 \\
\hline
\end{tabular}

Table 11: Detailed Results for the instance class B of Baldacci et al. (2007) 


\begin{tabular}{|c|c|c|c|c|c|c|c|c|c|}
\hline Instance & $|M|$ & $|K|$ & $U B$ & $L B$ & Time LP & Time IP & $\# \mathrm{~B} \& \mathrm{~B}$ & \#ExtNG & \#Cuts \\
\hline eil26.tsp.3.6.3.A.CEIL_2D & 25 & 6 & 178 & 178 & 0.1 & 0.1 & 1 & 0 & 0 \\
\hline eil26.tsp.3.12.5.A.CEIL_2D & 25 & 12 & 254 & 254 & 0.1 & 0.1 & 2 & 1 & 6 \\
\hline eil26.tsp.4.12.4.A.CEIL_2D & 25 & 12 & 271 & 271 & 0.1 & 0.1 & 1 & 0 & 0 \\
\hline eil26.tsp.5.12.3.A.CEIL_2D & 25 & 12 & 304 & 304 & 0.1 & 0.1 & 1 & 0 & 0 \\
\hline eil26.tsp.3.18.7.A.CEIL_2D & 25 & 18 & 312 & 312 & 0.1 & 0.4 & 10 & 7 & 48 \\
\hline eil26.tsp.4.18.5.A.CEIL_2D & 25 & 18 & 349 & 349 & 0.1 & 0.2 & 3 & 2 & 24 \\
\hline eil26.tsp.5.18.4.A.CEIL_2D & 25 & 18 & 387 & 387 & 0.1 & 0.1 & 3 & 2 & 40 \\
\hline eil26.tsp.7.18.3.A.CEIL_2D & 25 & 18 & 462 & 462 & 0.1 & 0.1 & 2 & 1 & 20 \\
\hline eil26.tsp.3.25.10.A.CEIL_2D & 25 & 25 & 346 & 346 & 0.1 & 0.8 & 12 & 8 & 56 \\
\hline eil26.tsp.4.25.7.A.CEIL_2D & 25 & 25 & 379 & 379 & 0.1 & 0.3 & 6 & 4 & 39 \\
\hline eil26.tsp.5.25.6.A.CEIL_2D & 25 & 25 & 398 & 398 & 0.1 & 0.1 & 1 & 0 & 0 \\
\hline eil26.tsp.7.25.4.A.CEIL_2D & 25 & 25 & 490 & 490 & 0.1 & 0.1 & 2 & 1 & 10 \\
\hline eil26.tsp.10.25.3.A.CEIL_2D & 25 & 25 & 601 & 601 & 0.1 & 0.1 & 4 & 3 & 37 \\
\hline eil51.tsp.3.12.5.A.CEIL_2D & 50 & 12 & 254 & 254 & 0.1 & 0.2 & 4 & 2 & 30 \\
\hline eil51.tsp.4.12.4.A.CEIL_2D & 50 & 12 & 271 & 271 & 0.1 & 0.1 & 2 & 1 & 3 \\
\hline eil51.tsp.5.12.3.A.CEIL_2D & 50 & 12 & 303 & 303 & 0.1 & 0.1 & 2 & 1 & 4 \\
\hline eil51.tsp.3.25.10.A.CEIL_2D & 50 & 25 & 343 & 343 & 0.4 & 2.0 & 10 & 6 & 65 \\
\hline eil51.tsp.4.25.7.A.CEIL_2D & 50 & 25 & 378 & 378 & 0.2 & 1.9 & 17 & 10 & 82 \\
\hline eil51.tsp.5.25.6.A.CEIL_2D & 50 & 25 & 395 & 395 & 0.1 & 0.2 & 1 & 0 & 0 \\
\hline eil51.tsp.7.25.4.A.CEIL_2D & 50 & 25 & 489 & 489 & 0.1 & 0.2 & 3 & 1 & 26 \\
\hline eil51.tsp.10.25.3.A.CEIL_2D & 50 & 25 & 595 & 595 & 0.1 & 0.2 & 2 & 1 & 49 \\
\hline eil51.tsp.3.37.14.A.CEIL_2D & 50 & 37 & 406 & 406 & 3.4 & 37.3 & 29 & 16 & 108 \\
\hline eil51.tsp.4.37.11.A.CEIL_2D & 50 & 37 & 437 & 437 & 0.9 & 28.7 & 29 & 20 & 115 \\
\hline eil51.tsp.5.37.9.A.CEIL_2D & 50 & 37 & 467 & 467 & 0.4 & 12.2 & 31 & 17 & 99 \\
\hline eil51.tsp.7.37.6.A.CEIL_2D & 50 & 37 & 547 & 547 & 0.3 & 2.4 & 15 & 11 & 97 \\
\hline eil51.tsp.10.37.5.A.CEIL_2D & 50 & 37 & 626 & 626 & 0.1 & 3.7 & 27 & 15 & 156 \\
\hline eil51.tsp.14.37.3.A.CEIL_2D & 50 & 37 & 829 & 829 & 0.1 & 1.2 & 12 & 7 & 122 \\
\hline eil51.tsp.3.50.19.A.CEIL_2D & 50 & 50 & - & 495 & 27.2 & 1800.0 & 77 & 40 & 110 \\
\hline eil51.tsp.4.50.14.A.CEIL_2D & 50 & 50 & 530 & 530 & 7.8 & 1075.4 & 100 & 61 & 116 \\
\hline eil51.tsp.5.50.12.A.CEIL_2D & 50 & 50 & 560 & 560 & 3.0 & 35.7 & 17 & 11 & 108 \\
\hline eil51.tsp.7.50.8.A.CEIL_2D & 50 & 50 & 648 & 648 & 1.2 & 67.6 & 66 & 34 & 162 \\
\hline eil51.tsp.10.50.6.A.CEIL_2D & 50 & 50 & 754 & 754 & 0.5 & 13.2 & 34 & 16 & 125 \\
\hline eil51.tsp.14.50.4.A.CEIL_2D & 50 & 50 & 954 & 954 & 0.2 & 5.9 & 29 & 10 & 234 \\
\hline
\end{tabular}

Table 12: Detailed Results for instance class small A of Hoshino and De Souza (2012)

\begin{tabular}{|c|c|c|c|c|c|c|c|c|c|}
\hline Instance & $|M|$ & $|K|$ & $U B$ & $L B$ & Time LP & Time IP & $\# B \& B$ & \#ExtNG & \#Cuts \\
\hline eil26.tsp.3.6.3.B.CEIL_2D & 25 & 6 & 1246 & 1246 & 0.1 & 0.1 & 1 & 0 & 0 \\
\hline eil26.tsp.3.12.5.B.CEIL_2D & 25 & 12 & 1778 & 1778 & 0.1 & 0.1 & 2 & 1 & 6 \\
\hline eil26.tsp.4.12.4.B.CEIL_2D & 25 & 12 & 1897 & 1897 & 0.1 & 0.1 & 1 & 0 & 0 \\
\hline eil26.tsp.5.12.3.B.CEIL_2D & 25 & 12 & 2128 & 2128 & 0.1 & 0.1 & 1 & 0 & 0 \\
\hline eil26.tsp.3.18.7.B.CEIL_2D & 25 & 18 & 2184 & 2184 & 0.1 & 0.3 & 10 & 5 & 50 \\
\hline eil26.tsp.4.18.5.B.CEIL_2D & 25 & 18 & 2443 & 2443 & 0.1 & 0.1 & 3 & 2 & 24 \\
\hline eil26.tsp.5.18.4.B.CEIL_2D & 25 & 18 & 2709 & 2709 & 0.1 & 0.1 & 3 & 2 & 40 \\
\hline eil26.tsp.7.18.3.B.CEIL_2D & 25 & 18 & 3234 & 3234 & 0.1 & 0.1 & 2 & 1 & 20 \\
\hline eil26.tsp.3.25.10.B.CEIL_2D & 25 & 25 & 2422 & 2422 & 0.1 & 0.7 & 12 & 8 & 58 \\
\hline eil26.tsp.4.25.7.B.CEIL_2D & 25 & 25 & 2653 & 2653 & 0.1 & 0.3 & 6 & 4 & 39 \\
\hline eil26.tsp.5.25.6.B.CEIL_2D & 25 & 25 & 2786 & 2786 & 0.1 & 0.1 & 1 & 0 & 0 \\
\hline eil26.tsp.7.25.4.B.CEIL_2D & 25 & 25 & 3430 & 3430 & 0.1 & 0.1 & 2 & 1 & 10 \\
\hline eil26.tsp.10.25.3.B.CEIL_2D & 25 & 25 & 4207 & 4207 & 0.1 & 0.1 & 4 & 3 & 37 \\
\hline eil51.tsp.3.12.5.B.CEIL_2D & 50 & 12 & 1778 & 1778 & 0.1 & 0.2 & 4 & 3 & 34 \\
\hline eil51.tsp.4.12.4.B.CEIL_2D & 50 & 12 & 1897 & 1897 & 0.1 & 0.1 & 2 & 1 & 3 \\
\hline eil51.tsp.5.12.3.B.CEIL_2D & 50 & 12 & 2121 & 2121 & 0.1 & 0.1 & 2 & 1 & 4 \\
\hline eil51.tsp.3.25.10.B.CEIL_2D & 50 & 25 & 2354 & 2354 & 0.4 & 1.4 & 7 & 4 & 56 \\
\hline eil51.tsp.4.25.7.B.CEIL_2D & 50 & 25 & 2606 & 2606 & 0.2 & 1.2 & 11 & 7 & 65 \\
\hline eil51.tsp.5.25.6.B.CEIL_2D & 50 & 25 & 2718 & 2718 & 0.1 & 0.1 & 1 & 0 & 0 \\
\hline eil51.tsp.7.25.4.B.CEIL_2D & 50 & 25 & 3400 & 3400 & 0.1 & 0.2 & 3 & 2 & 32 \\
\hline eil51.tsp.10.25.3.B.CEIL_2D & 50 & 25 & 4111 & 4111 & 0.1 & 0.1 & 2 & 1 & 26 \\
\hline eil51.tsp.3.37.14.B.CEIL_2D & 50 & 37 & 2795 & 2795 & 2.3 & 20.4 & 24 & 19 & 96 \\
\hline eil51.tsp.4.37.11.B.CEIL_2D & 50 & 37 & 3022 & 3022 & 0.6 & 45.7 & 52 & 35 & 116 \\
\hline eil51.tsp.5.37.9.B.CEIL_2D & 50 & 37 & 3236 & 3236 & 0.3 & 11.9 & 28 & 17 & 109 \\
\hline eil51.tsp.7.37.6.B.CEIL_2D & 50 & 37 & 3775 & 3775 & 0.3 & 0.7 & 5 & 3 & 61 \\
\hline eil51.tsp.10.37.5.B.CEIL_2D & 50 & 37 & 4335 & 4335 & 0.2 & 2.9 & 25 & 13 & 128 \\
\hline eil51.tsp.14.37.3.B.CEIL_2D & 50 & 37 & 5759 & 5759 & 0.1 & 1.0 & 10 & 7 & 137 \\
\hline eil51.tsp.3.50.19.B.CEIL_2D & 50 & 50 & - & 3385 & 34.4 & 1800.0 & 138 & 77 & 101 \\
\hline eil51.tsp.4.50.14.B.CEIL_2D & 50 & 50 & 3624 & 3624 & 6.4 & 617.2 & 90 & 40 & 88 \\
\hline eil51.tsp.5.50.12.B.CEIL_2D & 50 & 50 & 3853 & 3853 & 2.4 & 1770.0 & 426 & 146 & 127 \\
\hline eil51.tsp.7.50.8.B.CEIL_2D & 50 & 50 & 4474 & 4474 & 0.8 & 719.6 & 394 & 90 & 162 \\
\hline eil51.tsp.10.50.6.B.CEIL_2D & 50 & 50 & 5216 & 5216 & 0.5 & 4.8 & 20 & 11 & 83 \\
\hline eil51.tsp.14.50.4.B.CEIL_2D & 50 & 50 & 6612 & 6612 & 0.2 & 32.2 & 52 & 19 & 234 \\
\hline
\end{tabular}

Table 13: Detailed Results for instance class small B of Hoshino and De Souza (2012) 


\begin{tabular}{|c|c|c|c|c|c|c|c|c|c|}
\hline Instance & $|M|$ & $|K|$ & $U B$ & $L B$ & Time LP & Time IP & \#B\&B & \#ExtNG & \#Cuts \\
\hline eil26.tsp.3.6.3.C.CEIL_2D & 25 & 6 & 159 & 159 & 0.1 & 0.1 & 1 & 0 & 0 \\
\hline eil26.tsp.3.12.5.C.CEIL_2D & 25 & 12 & 226 & 226 & 0.1 & 0.1 & 1 & 0 & 0 \\
\hline eil26.tsp.4.12.4.C.CEIL_2D & 25 & 12 & 243 & 243 & 0.1 & 0.1 & 1 & 0 & 0 \\
\hline eil26.tsp.5.12.3.C.CEIL_2D & 25 & 12 & 270 & 270 & 0.1 & 0.1 & 3 & 0 & 1 \\
\hline eil26.tsp.3.18.7.C.CEIL_2D & 25 & 18 & 289 & 289 & 0.2 & 6.6 & 32 & 0 & 56 \\
\hline eil26.tsp.4.18.5.C.CEIL_2D & 25 & 18 & 324 & 324 & 0.1 & 0.2 & 3 & 0 & 0 \\
\hline eil26.tsp.5.18.4.C.CEIL_2D & 25 & 18 & 353 & 353 & 0.1 & 0.1 & 1 & 0 & 0 \\
\hline eil26.tsp.7.18.3.C.CEIL_2D & 25 & 18 & 410 & 410 & 0.1 & 0.1 & 1 & 0 & 0 \\
\hline eil26.tsp.3.25.10.C.CEIL_2D & 25 & 25 & 327 & 327 & 1.2 & 8.4 & 7 & 0 & 22 \\
\hline eil26.tsp.4.25.7.C.CEIL_2D & 25 & 25 & 362 & 362 & 0.2 & 1.7 & 12 & 0 & 28 \\
\hline eil26.tsp.5.25.6.C.CEIL_2D & 25 & 25 & 385 & 385 & 0.1 & 0.1 & 1 & 0 & 0 \\
\hline eil26.tsp.7.25.4.C.CEIL_2D & 25 & 25 & 460 & 460 & 0.1 & 0.1 & 1 & 0 & 0 \\
\hline eil26.tsp.10.25.3.C.CEIL_2D & 25 & 25 & 545 & 545 & 0.1 & 0.1 & 2 & 0 & 1 \\
\hline eil51.tsp.3.12.5.C.CEIL_2D & 50 & 12 & 226 & 226 & 0.1 & 0.1 & 1 & 0 & 0 \\
\hline eil51.tsp.4.12.4.C.CEIL_2D & 50 & 12 & 241 & 241 & 0.1 & 0.1 & 1 & 0 & 0 \\
\hline eil51.tsp.5.12.3.C.CEIL_2D & 50 & 12 & 270 & 270 & 0.1 & 0.2 & 5 & 0 & 0 \\
\hline eil51.tsp.3.25.10.C.CEIL_2D & 50 & 25 & 325 & 325 & 8.6 & 50.3 & 6 & 0 & 16 \\
\hline eil51.tsp.4.25.7.C.CEIL_2D & 50 & 25 & 359 & 359 & 0.7 & 14.4 & 24 & 0 & 73 \\
\hline eil51.tsp.5.25.6.C.CEIL_2D & 50 & 25 & 383 & 383 & 0.1 & 0.3 & 1 & 0 & 0 \\
\hline eil51.tsp.7.25.4.C.CEIL_2D & 50 & 25 & 457 & 457 & 0.1 & 0.1 & 1 & 0 & 0 \\
\hline eil51.tsp.10.25.3.C.CEIL_2D & 50 & 25 & 539 & 539 & 0.2 & 0.5 & 6 & 0 & 4 \\
\hline eil51.tsp.3.37.14.C.CEIL_2D & 50 & 37 & - & 394 & 1319.4 & 1800.0 & 1 & 0 & 21 \\
\hline eil51.tsp.4.37.11.C.CEIL_2D & 50 & 37 & - & 423 & 45.1 & 1800.0 & 19 & 0 & 72 \\
\hline eil51.tsp.5.37.9.C.CEIL_2D & 50 & 37 & 446 & 446 & 7.2 & 18.0 & 3 & 0 & 12 \\
\hline eil51.tsp.7.37.6.C.CEIL_2D & 50 & 37 & 530 & 530 & 0.7 & 10.7 & 24 & 0 & 45 \\
\hline eil51.tsp.10.37.5.C.CEIL_2D & 50 & 37 & 598 & 598 & 0.1 & 0.2 & 1 & 0 & 0 \\
\hline eil51.tsp.14.37.3.C.CEIL_2D & 50 & 37 & 765 & 765 & 0.2 & 1.7 & 20 & 0 & 7 \\
\hline eil51.tsp.3.50.19.C.CEIL_2D & 50 & 50 & - & - & 1800.0 & 1800.0 & 0 & 0 & 0 \\
\hline eil51.tsp.4.50.14.C.CEIL_2D & 50 & 50 & - & - & 1800.0 & 1800.0 & 0 & 0 & 0 \\
\hline eil51.tsp.5.50.12.C.CEIL_2D & 50 & 50 & - & 530 & 1014.0 & 1800.0 & 2 & 0 & 4 \\
\hline eil51.tsp.7.50.8.C.CEIL_2D & 50 & 50 & - & 620 & 5.0 & 1800.0 & 205 & 0 & 81 \\
\hline eil51.tsp.10.50.6.C.CEIL_2D & 50 & 50 & 721 & 721 & 0.9 & 8.7 & 17 & 0 & 42 \\
\hline eil51.tsp.14.50.4.C.CEIL_2D & 50 & 50 & 905 & 905 & 0.5 & 6.1 & 28 & 0 & 15 \\
\hline
\end{tabular}

Table 14: Detailed Results for instance class small C of Hoshino and De Souza (2012) 


\begin{tabular}{|c|c|c|c|c|c|c|c|c|c|}
\hline Instance & $|M|$ & $|K|$ & $U B$ & $L B$ & Time LP & Time IP & \#B\&B & \#ExtNG & \#Cuts \\
\hline eil76.tsp.3.18.7.A.CEIL_2D & 75 & 18 & 338 & 338 & 0.4 & 367.9 & 475 & 109 & 253 \\
\hline eil76.tsp.4.18.5.A.CEIL_2D & 75 & 18 & 399 & 399 & 0.2 & 17.5 & 86 & 15 & 184 \\
\hline eil76.tsp.5.18.4.A.CEIL_2D & 75 & 18 & 460 & 460 & 0.2 & 6.4 & 35 & 10 & 165 \\
\hline eil76.tsp.7.18.3.A.CEIL_2D & 75 & 18 & 558 & 558 & 0.1 & 0.3 & 4 & 3 & 12 \\
\hline eil76.tsp.3.37.14.A.CEIL_2D & 75 & 37 & - & 422 & 356.1 & 1800.0 & 5 & 4 & 40 \\
\hline eil76.tsp.4.37.11.A.CEIL_2D & 75 & 37 & - & 463 & 54.4 & 1800.0 & 22 & 13 & 149 \\
\hline eil76.tsp.5.37.9.A.CEIL_2D & 75 & 37 & 501 & 501 & 0.1 & 16.3 & 1 & 0 & 0 \\
\hline eil76.tsp.7.37.6.A.CEIL_2D & 75 & 37 & 641 & 641 & 2.1 & 3.2 & 2 & 1 & 1 \\
\hline eil76.tsp.10.37.5.A.CEIL_2D & 75 & 37 & 748 & 748 & 0.6 & 10.8 & 25 & 13 & 105 \\
\hline eil76.tsp.14.37.3.A.CEIL_2D & 75 & 37 & 1045 & 1045 & 0.3 & 1.6 & 6 & 3 & 39 \\
\hline eil76.tsp.3.56.21.A.CEIL_2D & 75 & 56 & — & — & 1800.0 & 1800.0 & 0 & 0 & 0 \\
\hline eil76.tsp.4.56.16.A.CEIL_2D & 75 & 56 & - & - & 1800.0 & 1800.0 & 0 & 0 & 0 \\
\hline eil76.tsp.5.56.13.A.CEIL_2D & 75 & 56 & - & - & 1800.0 & 1800.0 & 0 & 0 & 0 \\
\hline eil76.tsp.7.56.9.A.CEIL_2D & 75 & 56 & - & 703 & 40.9 & 1800.0 & 30 & 23 & 79 \\
\hline eil76.tsp.10.56.7.A.CEIL_2D & 75 & 56 & 824 & 824 & 3.6 & 67.0 & 21 & 12 & 91 \\
\hline eil76.tsp.14.56.5.A.CEIL_2D & 75 & 56 & 1030 & 1030 & 0.7 & 20.9 & 34 & 18 & 122 \\
\hline eil76.tsp.3.75.28.A.CEIL_2D & 75 & 75 & - & — & 1800.0 & 1800.0 & 0 & 0 & 0 \\
\hline eil76.tsp.4.75.21.A.CEIL_2D & 75 & 75 & - & - & 1800.0 & 1800.0 & 0 & 0 & 0 \\
\hline eil76.tsp.5.75.17.A.CEIL_2D & 75 & 75 & - & - & 1800.0 & 1800.0 & 0 & 0 & 0 \\
\hline eil76.tsp.7.75.12.A.CEIL_2D & 75 & 75 & - & - & 1800.0 & 1800.0 & 0 & 0 & 0 \\
\hline eil76.tsp.10.75.9.A.CEIL_2D & 75 & 75 & - & 931 & 152.5 & 1800.0 & 14 & 9 & 71 \\
\hline eil76.tsp.14.75.6.A.CEIL_2D & 75 & 75 & 1180 & 1180 & 4.1 & 71.0 & 31 & 15 & 115 \\
\hline eil101.tsp.3.25.10.A.CEIL_2D & 100 & 25 & 381 & 381 & 1.6 & 138.7 & 51 & 22 & 371 \\
\hline eil101.tsp.4.25.7.A.CEIL_2D & 100 & 25 & 433 & 433 & 0.9 & 50.9 & 62 & 22 & 356 \\
\hline eil101.tsp.5.25.6.A.CEIL_2D & 100 & 25 & 469 & 469 & 0.8 & 45.6 & 52 & 21 & 388 \\
\hline eil101.tsp.7.25.4.A.CEIL_2D & 100 & 25 & 576 & 576 & 0.6 & 124.7 & 209 & 38 & 383 \\
\hline eil101.tsp.10.25.3.A.CEIL_2D & 100 & 25 & 693 & 693 & 0.6 & 12.2 & 25 & 8 & 263 \\
\hline eil101.tsp.3.50.19.A.CEIL_2D & 100 & 50 & - & - & 1800.0 & 1800.0 & 0 & 0 & 0 \\
\hline eil101.tsp.4.50.14.A.CEIL_2D & 100 & 50 & - & - & 1800.0 & 1800.0 & 0 & 0 & 0 \\
\hline eil101.tsp.5.50.12.A.CEIL_2D & 100 & 50 & - & - & 1800.0 & 1800.0 & 0 & 0 & 0 \\
\hline eil101.tsp.7.50.8.A.CEIL_2D & 100 & 50 & - & 692 & 84.1 & 1800.0 & 25 & 9 & 136 \\
\hline eil101.tsp.10.50.6.A.CEIL_2D & 100 & 50 & 819 & 819 & 6.0 & 273.8 & 53 & 19 & 193 \\
\hline eil101.tsp.14.50.4.A.CEIL_2D & 100 & 50 & 1042 & 1042 & 1.7 & 517.1 & 198 & 39 & 392 \\
\hline eil101.tsp.3.75.28.A.CEIL_2D & 100 & 75 & - & - & 1800.0 & 1800.0 & 0 & 0 & 0 \\
\hline eil101.tsp.4.75.21.A.CEIL_2D & 100 & 75 & - & - & 1800.0 & 1800.0 & 0 & 0 & 0 \\
\hline eil101.tsp.5.75.17.A.CEIL_2D & 100 & 75 & - & - & 1800.0 & 1800.0 & 0 & 0 & 0 \\
\hline eil101.tsp.7.75.12.A.CEIL_2D & 100 & 75 & - & - & 1800.0 & 1800.0 & 0 & 0 & 0 \\
\hline eil101.tsp.10.75.9.A.CEIL_2D & 100 & 75 & - & 897 & 300.1 & 1800.0 & 6 & 4 & 51 \\
\hline eil101.tsp.14.75.6.A.CEIL_2D & 100 & 75 & - & 1126 & 10.1 & 1800.0 & 144 & 35 & 338 \\
\hline eil101.tsp.3.100.38.A.CEIL_2D & 100 & 100 & - & - & 1800.0 & 1800.0 & 0 & 0 & 0 \\
\hline eil101.tsp.4.100.28.A.CEIL_2D & 100 & 100 & - & - & 1800.0 & 1800.0 & 0 & 0 & 0 \\
\hline eil101.tsp.5.100.23.A.CEIL_2D & 100 & 100 & - & - & 1800.0 & 1800.0 & 0 & 0 & 0 \\
\hline eil101.tsp.7.100.16.A.CEIL_2D & 100 & 100 & - & - & 1800.0 & 1800.0 & 0 & 0 & 0 \\
\hline eil101.tsp.10.100.12.A.CEIL_2D & 100 & 100 & - & - & 1800.0 & 1800.0 & 0 & 0 & 0 \\
\hline eil101.tsp.14.100.8.A.CEIL_2D & 100 & 100 & - & 1176 & 96.0 & 1800.0 & 35 & 15 & 130 \\
\hline
\end{tabular}

Table 15: Detailed Results for instance class large A of Hoshino and De Souza (2012) 


\begin{tabular}{|c|c|c|c|c|c|c|c|c|c|}
\hline Instance & $|M|$ & $|K|$ & $U B$ & $L B$ & Time LP & Time IP & \#B\&B & \#ExtNG & \#Cuts \\
\hline eil76.tsp.3.18.7.B.CEIL_2D & 75 & 18 & 2319 & 2317 & 0.4 & 1800.0 & 2425 & 320 & 253 \\
\hline eil76.tsp.4.18.5.B.CEIL_2D & 75 & 18 & 2729 & 2729 & 0.2 & 10.7 & 53 & 9 & 177 \\
\hline eil76.tsp.5.18.4.B.CEIL_2D & 75 & 18 & 3172 & 3172 & 0.3 & 12.6 & 68 & 9 & 172 \\
\hline eil76.tsp.7.18.3.B.CEIL_2D & 75 & 18 & 3824 & 3824 & 0.1 & 0.2 & 3 & 2 & 31 \\
\hline eil76.tsp.3.37.14.B.CEIL_2D & 75 & 37 & - & 2906 & 342.3 & 1800.0 & 6 & 5 & 43 \\
\hline eil76.tsp.4.37.11.B.CEIL_2D & 75 & 37 & - & 3214 & 81.8 & 1800.0 & 28 & 13 & 145 \\
\hline eil76.tsp.5.37.9.B.CEIL_2D & 75 & 37 & 3495 & 3495 & 0.1 & 27.0 & 1 & 0 & 0 \\
\hline eil76.tsp.7.37.6.B.CEIL_2D & 75 & 37 & 4451 & 4451 & 2.0 & 4.5 & 3 & 2 & 12 \\
\hline eil76.tsp.10.37.5.B.CEIL_2D & 75 & 37 & 5177 & 5177 & 0.7 & 19.5 & 45 & 16 & 124 \\
\hline eil76.tsp.14.37.3.B.CEIL_2D & 75 & 37 & 7235 & 7235 & 0.3 & 7.2 & 82 & 16 & 95 \\
\hline eil76.tsp.3.56.21.B.CEIL_2D & 75 & 56 & - & - & 1800.0 & 1800.0 & 0 & 0 & 0 \\
\hline eil76.tsp.4.56.16.B.CEIL_2D & 75 & 56 & - & - & 1800.0 & 1800.0 & 0 & 0 & 0 \\
\hline eil76.tsp.5.56.13.B.CEIL_2D & 75 & 56 & - & - & 1800.0 & 1800.0 & 0 & 0 & 0 \\
\hline eil76.tsp.7.56.9.B.CEIL_2D & 75 & 56 & - & 4741 & 26.8 & 1800.0 & 49 & 29 & 112 \\
\hline eil76.tsp.10.56.7.B.CEIL_2D & 75 & 56 & 5541 & 5541 & 2.4 & 13.0 & 7 & 4 & 47 \\
\hline eil76.tsp.14.56.5.B.CEIL_2D & 75 & 56 & 7010 & 7010 & 0.7 & 800.2 & 721 & 144 & 178 \\
\hline eil76.tsp.3.75.28.B.CEIL_2D & 75 & 75 & - & - & 1800.0 & 1800.0 & 0 & 0 & 0 \\
\hline eil76.tsp.4.75.21.B.CEIL_2D & 75 & 75 & - & - & 1800.0 & 1800.0 & 0 & 0 & 0 \\
\hline eil76.tsp.5.75.17.B.CEIL_2D & 75 & 75 & - & - & 1800.0 & 1800.0 & 0 & 0 & 0 \\
\hline eil76.tsp.7.75.12.B.CEIL_2D & 75 & 75 & - & 5317 & 796.1 & 1800.0 & 2 & 2 & 12 \\
\hline eil76.tsp.10.75.9.B.CEIL_2D & 75 & 75 & - & 6241 & 20.0 & 1800.0 & 68 & 36 & 104 \\
\hline eil76.tsp.14.75.6.B.CEIL_2D & 75 & 75 & - & 8000 & 2.2 & 1800.0 & 506 & 217 & 177 \\
\hline eil101.tsp.3.25.10.B.CEIL_2D & 100 & 25 & 2629 & 2629 & 1.8 & 192.5 & 76 & 25 & 231 \\
\hline eil101.tsp.4.25.7.B.CEIL_2D & 100 & 25 & 2972 & 2972 & 0.9 & 20.8 & 31 & 15 & 157 \\
\hline eil101.tsp.5.25.6.B.CEIL_2D & 100 & 25 & 3237 & 3237 & 0.7 & 39.0 & 46 & 19 & 228 \\
\hline eil101.tsp.7.25.4.B.CEIL_2D & 100 & 25 & 3986 & 3986 & 0.5 & 194.4 & 537 & 56 & 291 \\
\hline eil101.tsp.10.25.3.B.CEIL_2D & 100 & 25 & 4803 & 4803 & 0.5 & 31.2 & 116 & 27 & 325 \\
\hline eil101.tsp.3.50.19.B.CEIL_2D & 100 & 50 & - & - & 1800.0 & 1800.0 & 0 & 0 & 0 \\
\hline eil101.tsp.4.50.14.B.CEIL_2D & 100 & 50 & - & - & 1800.0 & 1800.0 & 0 & 0 & 0 \\
\hline eil101.tsp.5.50.12.B.CEIL_2D & 100 & 50 & - & - & 1800.0 & 1800.0 & 0 & 0 & 0 \\
\hline eil101.tsp.7.50.8.B.CEIL_2D & 100 & 50 & - & 4729 & 28.4 & 1800.0 & 102 & 10 & 360 \\
\hline eil101.tsp.10.50.6.B.CEIL_2D & 100 & 50 & 5596 & 5596 & 5.1 & 771.1 & 226 & 34 & 180 \\
\hline eil101.tsp.14.50.4.B.CEIL_2D & 100 & 50 & 7130 & 7127 & 1.3 & 1800.0 & 647 & 81 & 422 \\
\hline eil101.tsp.3.75.28.B.CEIL_2D & 100 & 75 & - & - & 1800.0 & 1800.0 & 0 & 0 & 0 \\
\hline eil101.tsp.4.75.21.B.CEIL_2D & 100 & 75 & - & - & 1800.0 & 1800.0 & 0 & 0 & 0 \\
\hline eil101.tsp.5.75.17.B.CEIL_2D & 100 & 75 & - & - & 1800.0 & 1800.0 & 0 & 0 & 0 \\
\hline eil101.tsp.7.75.12.B.CEIL_2D & 100 & 75 & - & - & 1800.0 & 1800.0 & 0 & 0 & 0 \\
\hline eil101.tsp.10.75.9.B.CEIL_2D & 100 & 75 & 6012 & 6012 & 67.0 & 1192.9 & 32 & 6 & 144 \\
\hline eil101.tsp.14.75.6.B.CEIL_2D & 100 & 75 & 7530 & 7518 & 5.6 & 1800.0 & 376 & 126 & 323 \\
\hline eil101.tsp.3.100.38.B.CEIL_2D & 100 & 100 & - & - & 1800.0 & 1800.0 & 0 & 0 & 0 \\
\hline eil101.tsp.4.100.28.B.CEIL_2D & 100 & 100 & - & - & 1800.0 & 1800.0 & 0 & 0 & 0 \\
\hline eil101.tsp.5.100.23.B.CEIL_2D & 100 & 100 & - & - & 1800.0 & 1800.0 & 0 & 0 & 0 \\
\hline eil101.tsp.7.100.16.B.CEIL_2D & 100 & 100 & - & - & 1800.0 & 1800.0 & 0 & 0 & 0 \\
\hline eil101.tsp.10.100.12.B.CEIL_2D & 100 & 100 & - & 6404 & 694.3 & 1800.0 & 3 & 3 & 34 \\
\hline eil101.tsp.14.100.8.B.CEIL_2D & 100 & 100 & - & 7914 & 12.5 & 1800.0 & 171 & 57 & 182 \\
\hline
\end{tabular}

Table 16: Detailed Results for instance class large B of Hoshino and De Souza (2012) 


\begin{tabular}{|c|c|c|c|c|c|c|c|c|c|}
\hline Instance & $|M|$ & $|K|$ & $U B$ & $L B$ & Time LP & Time IP & \#B\&B & $\#$ ExtNG & \#Cuts \\
\hline eil76.tsp.3.18.7.C.CEIL_2D & 75 & 18 & - & 324 & 5.1 & 1800.0 & 123 & 0 & 404 \\
\hline eil76.tsp.4.18.5.C.CEIL_2D & 75 & 18 & 385 & 385 & 0.5 & 22.2 & 47 & 2 & 367 \\
\hline eil76.tsp.5.18.4.C.CEIL_2D & 75 & 18 & 439 & 439 & 0.4 & 30.1 & 70 & 5 & 232 \\
\hline eil76.tsp.7.18.3.C.CEIL_2D & 75 & 18 & 527 & 527 & 0.2 & 1.6 & 19 & 0 & 12 \\
\hline eil76.tsp.3.37.14.C.CEIL_2D & 75 & 37 & - & - & 1800.0 & 1800.0 & 0 & 0 & 0 \\
\hline eil76.tsp.4.37.11.C.CEIL_2D & 75 & 37 & - & - & 1800.0 & 1800.0 & 0 & 0 & 0 \\
\hline eil76.tsp.5.37.9.C.CEIL_2D & 75 & 37 & 491 & 491 & 46.1 & 86.0 & 2 & 0 & 2 \\
\hline eil76.tsp.7.37.6.C.CEIL_2D & 75 & 37 & 626 & 626 & 2.1 & 145.9 & 93 & 0 & 87 \\
\hline eil76.tsp.10.37.5.C.CEIL_2D & 75 & 37 & 723 & 723 & 1.2 & 20.8 & 28 & 3 & 45 \\
\hline eil76.tsp.14.37.3.C.CEIL_2D & 75 & 37 & 969 & 969 & 0.7 & 2.1 & 4 & 0 & 19 \\
\hline eil76.tsp.3.56.21.C.CEIL_2D & 75 & 56 & - & - & 1800.0 & 1800.0 & 0 & 0 & 0 \\
\hline eil76.tsp.4.56.16.C.CEIL_2D & 75 & 56 & - & - & 1800.0 & 1800.0 & 0 & 0 & 0 \\
\hline eil76.tsp.5.56.13.C.CEIL_2D & 75 & 56 & - & - & 1800.0 & 1800.0 & 0 & 0 & 0 \\
\hline eil76.tsp.7.56.9.C.CEIL_2D & 75 & 56 & - & 683 & 60.9 & 1800.0 & 18 & 1 & 70 \\
\hline eil76.tsp.10.56.7.C.CEIL_2D & 75 & 56 & 811 & 805 & 4.3 & 1800.0 & 335 & 0 & 95 \\
\hline eil76.tsp.14.56.5.C.CEIL_2D & 75 & 56 & 1000 & 1000 & 2.0 & 105.2 & 339 & 1 & 79 \\
\hline eil76.tsp.3.75.28.C.CEIL_2D & 75 & 75 & - & - & 1800.0 & 1800.0 & 0 & 0 & 0 \\
\hline eil76.tsp.4.75.21.C.CEIL_2D & 75 & 75 & - & - & 1800.0 & 1800.0 & 0 & 0 & 0 \\
\hline eil76.tsp.5.75.17.C.CEIL_2D & 75 & 75 & - & - & 1800.0 & 1800.0 & 0 & 0 & 0 \\
\hline eil76.tsp.7.75.12.C.CEIL_2D & 75 & 75 & - & - & 1800.0 & 1800.0 & 0 & 0 & 0 \\
\hline eil76.tsp.10.75.9.C.CEIL_2D & 75 & 75 & - & 903 & 132.0 & 1800.0 & 14 & 0 & 77 \\
\hline eil76.tsp.14.75.6.C.CEIL_2D & 75 & 75 & 1145 & 1145 & 3.6 & 58.2 & 25 & 1 & 79 \\
\hline eil101.tsp.3.25.10.C.CEIL_2D & 100 & 25 & - & 367 & 454.9 & 1800.0 & 3 & 0 & 35 \\
\hline eil101.tsp.4.25.7.C.CEIL_2D & 100 & 25 & 416 & 416 & 4.3 & 448.0 & 106 & 0 & 374 \\
\hline eil101.tsp.5.25.6.C.CEIL_2D & 100 & 25 & 440 & 440 & 2.8 & 8.1 & 4 & 0 & 10 \\
\hline eil101.tsp.7.25.4.C.CEIL_2D & 100 & 25 & 542 & 542 & 1.2 & 419.9 & 227 & 3 & 354 \\
\hline eil101.tsp.10.25.3.C.CEIL_2D & 100 & 25 & 631 & 631 & 1.0 & 3.9 & 5 & 1 & 5 \\
\hline eil101.tsp.3.50.19.C.CEIL_2D & 100 & 50 & - & - & 1800.0 & 1800.0 & 0 & 0 & 0 \\
\hline eil101.tsp.4.50.14.C.CEIL_2D & 100 & 50 & - & - & 1800.0 & 1800.0 & 0 & 0 & 0 \\
\hline eil101.tsp.5.50.12.C.CEIL_2D & 100 & 50 & - & - & 1800.0 & 1800.0 & 0 & 0 & 0 \\
\hline eil101.tsp.7.50.8.C.CEIL_2D & 100 & 50 & - & 671 & 29.4 & 1800.0 & 46 & 1 & 121 \\
\hline eil101.tsp.10.50.6.C.CEIL_2D & 100 & 50 & 793 & 793 & 5.9 & 179.7 & 58 & 0 & 97 \\
\hline eil101.tsp.14.50.4.C.CEIL_2D & 100 & 50 & 993 & 993 & 3.8 & 7.2 & 3 & 0 & 11 \\
\hline eil101.tsp.3.75.28.C.CEIL_2D & 100 & 75 & - & - & 1800.0 & 1800.0 & 0 & 0 & 0 \\
\hline eil101.tsp.4.75.21.C.CEIL_2D & 100 & 75 & - & - & 1800.0 & 1800.0 & 0 & 0 & 0 \\
\hline eil101.tsp.5.75.17.C.CEIL_2D & 100 & 75 & - & - & 1800.0 & 1800.0 & 0 & 0 & 0 \\
\hline eil101.tsp.7.75.12.C.CEIL_2D & 100 & 75 & - & - & 1800.0 & 1800.0 & 0 & 0 & 0 \\
\hline eil101.tsp.10.75.9.C.CEIL_2D & 100 & 75 & - & 876 & 327.7 & 1800.0 & 11 & 1 & 73 \\
\hline eil101.tsp.14.75.6.C.CEIL_2D & 100 & 75 & - & 1096 & 9.5 & 1800.0 & 769 & 1 & 122 \\
\hline eil101.tsp.3.100.38.C.CEIL_2D & 100 & 100 & - & - & 1800.0 & 1800.0 & 0 & 0 & 0 \\
\hline eil101.tsp.4.100.28.C.CEIL_2D & 100 & 100 & - & - & 1800.0 & 1800.0 & 0 & 0 & 0 \\
\hline eil101.tsp.5.100.23.C.CEIL_2D & 100 & 100 & - & - & 1800.0 & 1800.0 & 0 & 0 & 0 \\
\hline eil101.tsp.7.100.16.C.CEIL_2D & 100 & 100 & - & - & 1800.0 & 1800.0 & 0 & 0 & 0 \\
\hline eil101.tsp.10.100.12.C.CEIL_2D & 100 & 100 & - & - & 1800.0 & 1800.0 & 0 & 0 & 0 \\
\hline eil101.tsp.14.100.8.C.CEIL_2D & 100 & 100 & - & 1147 & 42.0 & 1800.0 & 68 & 0 & 86 \\
\hline
\end{tabular}

Table 17: Detailed Results for instance class large C of Hoshino and De Souza (2012) 


\begin{tabular}{|c|c|c|c|c|c|c|c|c|c|c|c|}
\hline Instance & $|M|+1$ & $|K|$ & $f r$ & $\# S u b$ & $U B$ & $L B$ & Time LP & Time IP & \#B\&B & \#ExtNG & \#Cuts \\
\hline CapEuclideo.20.10.30.5 & 20 & 10 & 30 & 4 & 3286 & 3286 & 0.1 & 0.1 & 1 & 0 & 0 \\
\hline CapEuclideo.20.10.30.2 & 20 & 10 & 30 & 4 & 4267 & 4267 & 0.1 & 0.1 & 9 & 0 & 0 \\
\hline CapEuclideo.20.10.55.5 & 20 & 10 & 55 & 4 & 2784 & 2784 & 0.1 & 0.1 & 1 & 0 & 0 \\
\hline CapEuclideo.20.10.55.2 & 20 & 10 & 55 & 4 & 4036 & 4036 & 0.1 & 0.1 & 1 & 0 & 0 \\
\hline CapEuclideo.20.10.80.5 & 20 & 10 & 80 & 2 & 2422 & 2422 & 0.1 & 0.1 & 1 & 0 & 0 \\
\hline CapEuclideo.20.10.80.2 & 20 & 10 & 80 & 2 & 3928 & 3928 & 0.1 & 0.1 & 19 & 0 & 0 \\
\hline CapEuclideo.20.30.30.15 & 20 & 30 & 30 & 35 & 8419 & 8419 & 1.5 & 1.5 & 1 & 0 & 0 \\
\hline CapEuclideo.20.30.30.5 & 20 & 30 & 30 & 35 & 8419 & 8419 & 0.5 & 0.5 & 1 & 0 & 0 \\
\hline CapEuclideo.20.30.55.15 & 20 & 30 & 55 & 16 & 6359 & 6359 & 1.1 & 1.1 & 1 & 0 & 0 \\
\hline CapEuclideo.20.30.55.3 & 20 & 30 & 55 & 16 & 7899 & 7899 & 0.1 & 1.4 & 31 & 0 & 0 \\
\hline CapEuclideo.20.30.80.15 & 20 & 30 & 80 & 5 & 4655 & 4655 & 2.3 & 4.9 & 3 & 0 & 0 \\
\hline CapEuclideo.20.30.80.2 & 20 & 30 & 80 & 5 & 10521 & 10521 & 0.1 & 0.5 & 41 & 0 & 0 \\
\hline CapEuclideo.20.50.30.25 & 20 & 50 & 30 & 102 & 11264 & 11264 & 11.2 & 126.2 & 43 & 3 & 0 \\
\hline CapEuclideo.20.50.30.9 & 20 & 50 & 30 & 102 & 11264 & 11264 & 9.5 & 106.9 & 45 & 2 & 0 \\
\hline CapEuclideo.20.50.55.17 & 20 & 50 & 55 & 35 & 9392 & 9392 & 10.8 & 15.5 & 3 & 0 & 0 \\
\hline CapEuclideo.20.50.55.4 & 20 & 50 & 55 & 35 & 10523 & 10523 & 1.2 & 60.7 & 144 & 1 & 0 \\
\hline CapEuclideo.20.50.80.25 & 20 & 50 & 80 & 11 & 6181 & 6181 & 16.4 & 16.4 & 1 & 0 & 0 \\
\hline CapEuclideo.20.50.80.4 & 20 & 50 & 80 & 11 & 9613 & 9613 & 0.3 & 18.9 & 162 & 5 & 0 \\
\hline CapEuclideo.20.70.30.18 & 20 & 70 & 30 & 286 & 19460 & 19460 & 75.5 & 75.5 & 1 & 0 & 0 \\
\hline CapEuclideo.20.70.30.6 & 20 & 70 & 30 & 286 & 19536 & 19536 & 25.4 & 25.4 & 1 & 0 & 0 \\
\hline CapEuclideo.20.70.55.35 & 20 & 70 & 55 & 87 & 14133 & 14133 & 139.9 & 144.0 & 2 & 0 & 0 \\
\hline CapEuclideo.20.70.55.7 & 20 & 70 & 55 & 87 & 14209 & 14209 & 14.2 & 72.7 & 15 & 0 & 0 \\
\hline CapEuclideo.20.70.80.35 & 20 & 70 & 80 & 12 & 9480 & 9480 & 53.4 & 53.4 & 1 & 0 & 0 \\
\hline CapEuclideo.20.70.80.5 & 20 & 70 & 80 & 12 & 13086 & 13085 & 0.7 & 21.1 & 67 & 2 & 0 \\
\hline CapEuclideo.35.10.30.5 & 35 & 10 & 30 & 5 & 1351 & 1351 & 0.1 & 0.1 & 1 & 0 & 0 \\
\hline CapEuclideo.35.10.30.2 & 35 & 10 & 30 & 5 & 1596 & 1596 & 0.1 & 0.1 & 3 & 0 & 0 \\
\hline CapEuclideo.35.10.55.4 & 35 & 10 & 55 & 4 & 1342 & 1342 & 0.1 & 0.1 & 1 & 0 & 0 \\
\hline CapEuclideo.35.10.55.2 & 35 & 10 & 55 & 4 & 1538 & 1538 & 0.1 & 0.1 & 3 & 0 & 0 \\
\hline CapEuclideo.35.10.80.5 & 35 & 10 & 80 & 2 & 1187 & 1187 & 0.1 & 0.1 & 1 & 0 & 0 \\
\hline CapEuclideo.35.10.80.2 & 35 & 10 & 80 & 2 & 1538 & 1538 & 0.1 & 0.1 & 7 & 0 & 0 \\
\hline CapEuclideo.35.30.30.10 & 35 & 30 & 30 & 25 & 5236 & 5236 & 1.0 & 1.0 & 1 & 0 & 0 \\
\hline CapEuclideo.35.30.30.4 & 35 & 30 & 30 & 25 & 5245 & 5245 & 0.5 & 1.1 & 3 & 0 & 0 \\
\hline CapEuclideo.35.30.55.15 & 35 & 30 & 55 & 15 & 4097 & 4097 & 4.7 & 4.7 & 1 & 0 & 0 \\
\hline CapEuclideo.35.30.55.3 & 35 & 30 & 55 & 15 & 4703 & 4703 & 0.1 & 0.5 & 7 & 0 & 0 \\
\hline CapEuclideo.35.30.80.8 & 35 & 30 & 80 & 5 & 3501 & 3501 & 1.3 & 10.8 & 12 & 2 & 0 \\
\hline CapEuclideo.35.30.80.2 & 35 & 30 & 80 & 5 & 5713 & 5713 & 0.1 & 4.5 & 135 & 0 & 0 \\
\hline CapEuclideo.35.50.30.17 & 35 & 50 & 30 & 62 & 12907 & 12907 & 25.7 & 25.7 & 1 & 0 & 0 \\
\hline CapEuclideo.35.50.30.6 & 35 & 50 & 30 & 62 & 12907 & 12907 & 9.1 & 9.1 & 1 & 0 & 0 \\
\hline CapEuclideo.35.50.55.13 & 35 & 50 & 55 & 45 & 9324 & 9324 & 75.2 & 75.2 & 1 & 0 & 0 \\
\hline CapEuclideo.35.50.55.3 & 35 & 50 & 55 & 45 & 11160 & 11160 & 0.8 & 7.2 & 27 & 0 & 0 \\
\hline CapEuclideo.35.50.80.25 & 35 & 50 & 80 & 11 & 5640 & 5640 & 1508.3 & 1508.3 & 1 & 0 & 0 \\
\hline CapEuclideo.35.50.80.4 & 35 & 50 & 80 & 11 & 8723 & 8723 & 0.8 & 4.4 & 23 & 0 & 0 \\
\hline CapEuclideo.35.70.30.18 & 35 & 70 & 30 & 147 & 12133 & 12133 & 47.2 & 47.2 & 1 & 0 & 0 \\
\hline CapEuclideo.35.70.30.6 & 35 & 70 & 30 & 147 & 12308 & 12308 & 21.8 & 59.5 & 9 & 0 & 0 \\
\hline CapEuclideo.35.70.55.24 & 35 & 70 & 55 & 81 & 9521 & 9521 & 99.0 & 99.0 & 1 & 0 & 0 \\
\hline CapEuclideo.35.70.55.5 & 35 & 70 & 55 & 81 & 10195 & 10195 & 10.1 & 62.6 & 13 & 0 & 0 \\
\hline CapEuclideo.35.70.80.35 & 35 & 70 & 80 & 15 & 6911 & 6911 & 152.0 & 152.0 & 1 & 0 & 0 \\
\hline CapEuclideo.35.70.80.5 & 35 & 70 & 80 & 15 & 8944 & 8944 & 2.0 & 158.5 & 241 & 11 & 0 \\
\hline CapEuclideo.50.10.30.4 & 50 & 10 & 30 & 6 & 2869 & 2869 & 0.1 & 0.1 & 1 & 0 & 0 \\
\hline CapEuclideo.50.10.30.2 & 50 & 10 & 30 & 6 & 3045 & 3045 & 0.1 & 0.1 & 1 & 0 & 0 \\
\hline CapEuclideo.50.10.55.4 & 50 & 10 & 55 & 4 & 2371 & 2371 & 0.1 & 0.1 & 1 & 0 & 0 \\
\hline CapEuclideo.50.10.55.2 & 50 & 10 & 55 & 4 & 2803 & 2803 & 0.1 & 0.1 & 1 & 0 & 0 \\
\hline CapEuclideo.50.10.80.5 & 50 & 10 & 80 & 2 & 1779 & 1779 & 0.2 & 0.2 & 1 & 0 & 0 \\
\hline CapEuclideo.50.10.80.2 & 50 & 10 & 80 & 2 & 2803 & 2803 & 0.1 & 0.1 & 1 & 0 & 0 \\
\hline CapEuclideo.50.30.30.8 & 50 & 30 & 30 & 33 & 5708 & 5708 & 10.5 & 10.5 & 1 & 0 & 0 \\
\hline CapEuclideo.50.30.30.3 & 50 & 30 & 30 & 33 & 5803 & 5803 & 0.5 & 0.5 & 1 & 0 & 0 \\
\hline CapEuclideo.50.30.55.15 & 50 & 30 & 55 & 15 & 5008 & 5008 & 32.8 & 32.8 & 1 & 0 & 0 \\
\hline CapEuclideo.50.30.55.3 & 50 & 30 & 55 & 15 & 5487 & 5487 & 0.3 & 0.8 & 5 & 0 & 0 \\
\hline CapEuclideo.50.30.80.15 & 50 & 30 & 80 & 5 & 3601 & 3601 & 235.3 & 235.3 & 1 & 0 & 0 \\
\hline CapEuclideo.50.30.80.2 & 50 & 30 & 80 & 5 & 6448 & 6448 & 0.1 & 0.3 & 5 & 0 & 0 \\
\hline CapEuclideo.50.50.30.25 & 50 & 50 & 30 & 138 & 7919 & 7919 & 52.7 & 52.7 & 1 & 0 & 0 \\
\hline CapEuclideo.50.50.30.9 & 50 & 50 & 30 & 138 & 7919 & 7919 & 44.1 & 44.1 & 1 & 0 & 0 \\
\hline CapEuclideo.50.50.55.25 & 50 & 50 & 55 & 37 & 6807 & 6807 & 66.5 & 66.5 & 1 & 0 & 0 \\
\hline CapEuclideo.50.50.55.5 & 50 & 50 & 55 & 37 & 7271 & 7271 & 4.9 & 101.5 & 47 & 0 & 0 \\
\hline CapEuclideo.50.50.80.17 & 50 & 50 & 80 & 9 & 5222 & 5222 & 28.1 & 798.2 & 15 & 0 & 0 \\
\hline CapEuclideo.50.50.80.3 & 50 & 50 & 80 & 9 & 8425 & 8425 & 0.3 & 17.6 & 135 & 0 & 0 \\
\hline CapEuclideo.50.70.30.35 & 50 & 70 & 30 & 237 & 10132 & 10132 & 283.7 & 2941.0 & 17 & 0 & 0 \\
\hline CapEuclideo.50.70.30.12 & 50 & 70 & 30 & 237 & 10140 & 10140 & 251.8 & 1904.9 & 15 & 0 & 0 \\
\hline CapEuclideo.50.70.55.24 & 50 & 70 & 55 & 68 & 9167 & 9167 & 451.6 & 615.3 & 2 & 0 & 0 \\
\hline CapEuclideo.50.70.55.5 & 50 & 70 & 55 & 68 & 9725 & 9725 & 13.5 & 37.1 & 7 & 0 & 0 \\
\hline CapEuclideo.50.70.80.35 & 50 & 70 & 80 & 17 & - & - & 7200.0 & 7200.0 & 0 & 0 & 0 \\
\hline CapEuclideo.50.70.80.5 & 50 & 70 & 80 & 17 & 8360 & 8360 & 3.3 & 7095.1 & 3198 & 511 & 0 \\
\hline
\end{tabular}

Table 18: Detailed Results for the instances of Gendreau et al. (2016) 